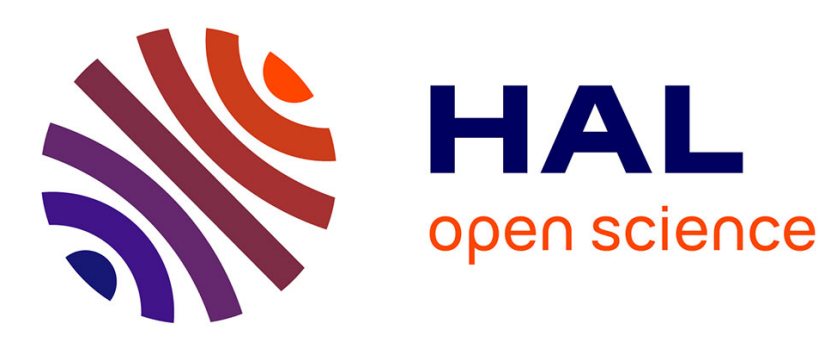

\title{
Stability of a swirled liquid film entrained by a fast gas stream
}

Jean-Philippe Matas, Moongeun Hong, Alain H. Cartellier

\section{To cite this version:}

Jean-Philippe Matas, Moongeun Hong, Alain H. Cartellier. Stability of a swirled liquid film entrained by a fast gas stream. Physics of Fluids, 2014, 26, pp.042108. hal-00987576

\section{HAL Id: hal-00987576 https://hal.science/hal-00987576}

Submitted on 6 May 2014

HAL is a multi-disciplinary open access archive for the deposit and dissemination of scientific research documents, whether they are published or not. The documents may come from teaching and research institutions in France or abroad, or from public or private research centers.
L'archive ouverte pluridisciplinaire HAL, est destinée au dépôt et à la diffusion de documents scientifiques de niveau recherche, publiés ou non, émanant des établissements d'enseignement et de recherche français ou étrangers, des laboratoires publics ou privés. 


\section{AIP $\mid$ Physics of Fluids}

\section{Stability of a swirled liquid film entrained by a fast gas stream}

Jean-Philippe Matas, Moongeun Hong, and Alain Cartellier

Citation: Physics of Fluids (1994-present) 26, 042108 (2014); doi: 10.1063/1.4871395

View online: http://dx.doi.org/10.1063/1.4871395

View Table of Contents: http://scitation.aip.org/content/aip/journal/pof2/26/4?ver=pdfcov

Published by the AIP Publishing

\section{Articles you may be interested in}

An experimental investigation of fingering instabilities and growth dynamics in inclined counter-current gas-liquid channel flow

Phys. Fluids 25, 122104 (2013); 10.1063/1.4851135

Two-wave structure of liquid film and wave interrelation in annular gas-liquid flow with and without entrainment

Phys. Fluids 21, 061701 (2009); 10.1063/1.3151999

Nonlinear breakup of a coaxial liquid jet in a swirling gas stream

Phys. Fluids 18, 114101 (2006); 10.1063/1.2364262

Thermal radiation and thickness fluctuations in freely suspended liquid films

Phys. Fluids 18, 085110 (2006); 10.1063/1.2337997

Temporal instability of swirling gas jets injected in liquids

Phys. Fluids 13, 2845 (2001); 10.1063/1.1396670

\section{AlP Re-register for Table of Content Alerts}




\title{
Stability of a swirled liquid film entrained by a fast gas stream
}

\author{
Jean-Philippe Matas, ${ }^{1, a)}$ Moongeun Hong, ${ }^{2}$ and Alain Cartellier ${ }^{1}$ \\ ${ }^{1}$ Université Grenoble Alpes, LEGI, F-38000 Grenoble, France and CNRS, LEGI, \\ F-38000 Grenoble, France \\ ${ }^{2}$ Korea Aerospace Research Institute, Daejeon 305-806, South Korea and Korea University of \\ Science and Technology, Daejeon 305-350, South Korea
}

(Received 5 November 2013; accepted 31 March 2014; published online 28 April 2014)

\begin{abstract}
We study the liquid flow inside a recessed gas-centered swirl coaxial injector, where a swirled liquid flowing against an outer wall is destabilized by a central fast gas stream. We present measurements of the liquid intact length inside the injector, as a function of swirl number and dynamic pressure ratio. We propose a simple model to account for the effect of these parameters. We next study the surface instability inside the injector: its frequency is measured for several swirl angles, and as a function of gas velocity. Results are first confronted to the predictions of an inviscid linear stability analysis including swirl, and second to the predictions of a viscous linear stability analysis where swirl is not included. The viscous analysis captures the experimental frequency. @ 2014 AIP Publishing LLC. [http://dx.doi.org/10.1063/1.4871395]
\end{abstract}

\section{INTRODUCTION}

The break-up of a liquid bulk into droplets is the goal of a wide range of industrial applications, and for the last decades a considerable amount of study has been conducted to understand the mechanisms behind various atomization devices. ${ }^{1}$ Notably, atomization phenomena in applications related to liquid rocket engines have been actively studied, in order to improve injection systems and thereby optimize combustion. However, various aspects of atomization characteristics of twophase coaxial injectors, which are usually used for liquid rocket engines, are still not sufficiently understood. Moreover, most of the related studies have been focused on the atomization of a liquid jet or sheet by a cocurrent gas flow. There are few studies on atomization of a swirled liquid film with one free and one wall-bounded surface. This configuration is relevant to liquid hydrocarbon rocket engines with a staged combustion cycle for high-power applications: a swirled liquid hydrocarbon film is stripped and fragmented by a high velocity oxygen gas flow in so-called Gas-Centered Swirl Coaxial (GCSC) injectors. However, available results on atomization characteristics in this injection configuration are too scarce to be helpful for design. Previous works have shown that the dynamic pressure ratio, recess length, and swirl strength are the main parameters determining the overall spray characteristics of GCSC injectors (Lightfoot $e t a l^{2-4}$ ). For example, Jeon et al..$^{5}$ proposed a critical dynamic pressure ratio to identify regimes of internal or external mixing in the injectors. Regarding the interfacial instability, Harper et al. ${ }^{6}$ performed a numerical analysis to determine the unsteady hydrodynamic characteristics inside GCSC injectors and showed that the liquid interfacial frequency decreases as liquid film thickness and swirl velocity increase. Recently, Schumaker et $l_{.}{ }^{7}$ studied the effect of swirl strength on the intact liquid film length inside the injectors. They showed that the liquid film length without liquid swirl is shorter than any of the cases with swirl when the total momentum flux ratio is less than 50. Moreover, they suggested the total momentum flux ratio could be used to scale the intact film length, and ultimately the atomization ratio and efficiency. In order to improve the understanding of atomization in GCSC injectors, we carry out measurements on the two-phase flow inside the injectors. The effect of swirl strength on the intact liquid length is

a)Electronic mail: matas@legi.grenoble-inp.fr 
investigated by internal flow visualization. We propose a simple model based on energy conservation to account for these experimental results. In addition, stability of the interface between the fast gas stream and the swirled liquid film is investigated via spectral measurement of the most unstable mode, and experimental results are confronted to predictions from inviscid and viscous stability analyses.

We detail the experimental setup in Sec. II. We next present experimental results, namely, measurements of liquid intact length, frequency, and wave amplitude. Section IV introduces our model for the liquid intact length, and compares it to experimental results. Section V is devoted to an inviscid stability analysis, and to the comparison of its results with experimental frequencies. Finally, Sec. VI presents the viscous stability analysis, and comparison with results plus discussion.

\section{EXPERIMENTAL SETUP AND METHODS}

Figure 1 shows a sketch of the GCSC injector used in the present study. In applications related to liquid rocket engines, the gaseous propellant (GOX) enters at a high velocity directly through the center of the injector. The swirled liquid propellant film (Kerosene) is injected along the periphery of the injection element, see Figure 1, and then stripped and fragmented into drops by the high velocity gas stream. In the present study, we use water and air at atmospheric pressure.

The injectors are made of plexiglass. They have a square outer cross-section in order to facilitate visualization of the internal flow, and to limit distortion due to refraction. Different diameters $d_{g}$ for gas flow at the end of sheltering lip are investigated, namely, 10.4 and $10.8 \mathrm{~mm}$. The outer diameter $d_{o}$ is $12.4 \mathrm{~mm}$ and the lip thickness is $0.3 \mathrm{~mm}$. Consequently, the liquid channel thickness $H_{l}$ becomes, respectively, 0.5 and $0.7 \mathrm{~mm}$ for $d_{g}=10.8$ and $10.4 \mathrm{~mm}$, respectively. The diameter of the four liquid tangential entries $d_{t}$ is $1.0 \pm 0.01 \mathrm{~mm}$. The recess length $l_{R}$ is fixed to $11.4 \mathrm{~mm}$ (corresponding to a recess ratio $l_{R} / d_{o}=0.92$ ). The volumetric liquid flow rate is measured with an oval wheel flow meter (OVAL). Air is supplied by laboratory compressed air, and its flow rate measured with a mass flow meter (Brooks Instruments). The uncertainty on the measured liquid and
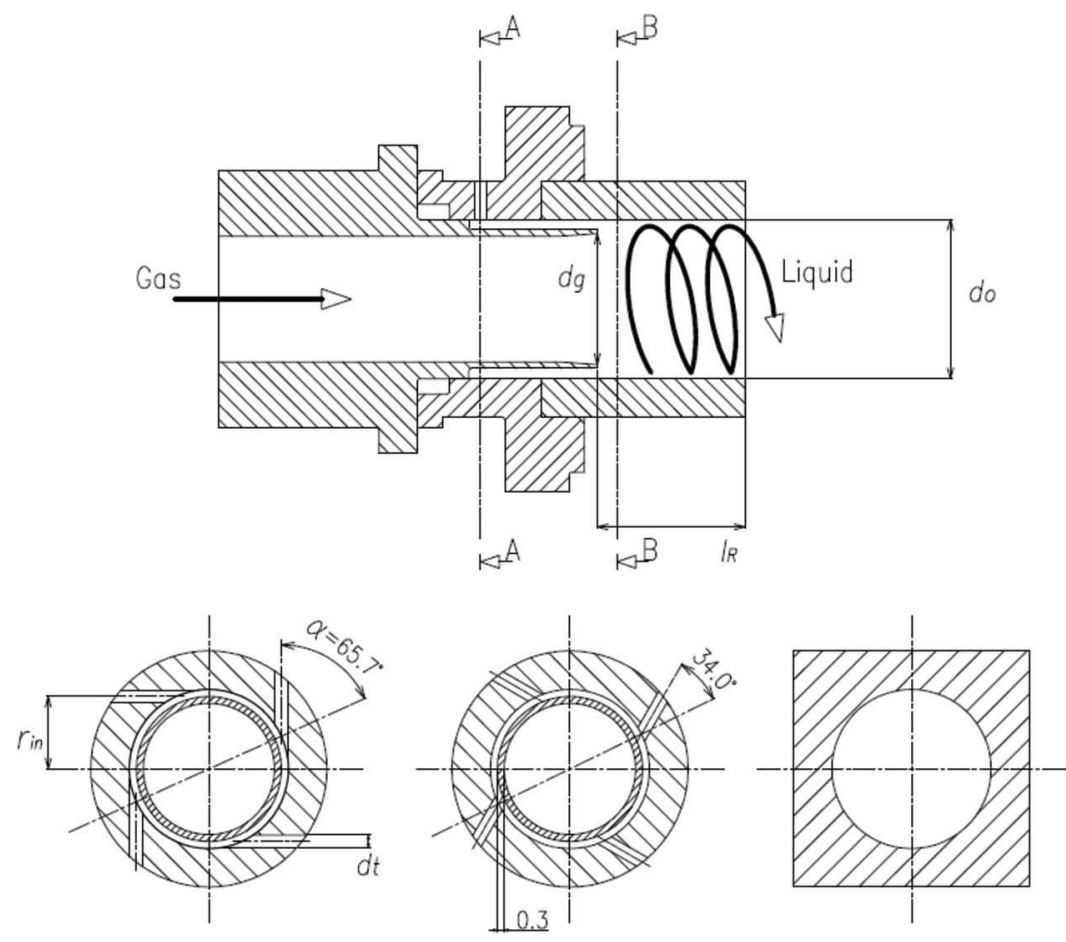

SECTION A-A

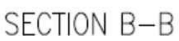

FIG. 1. Sketch of the GCSC injector. 
$\alpha$

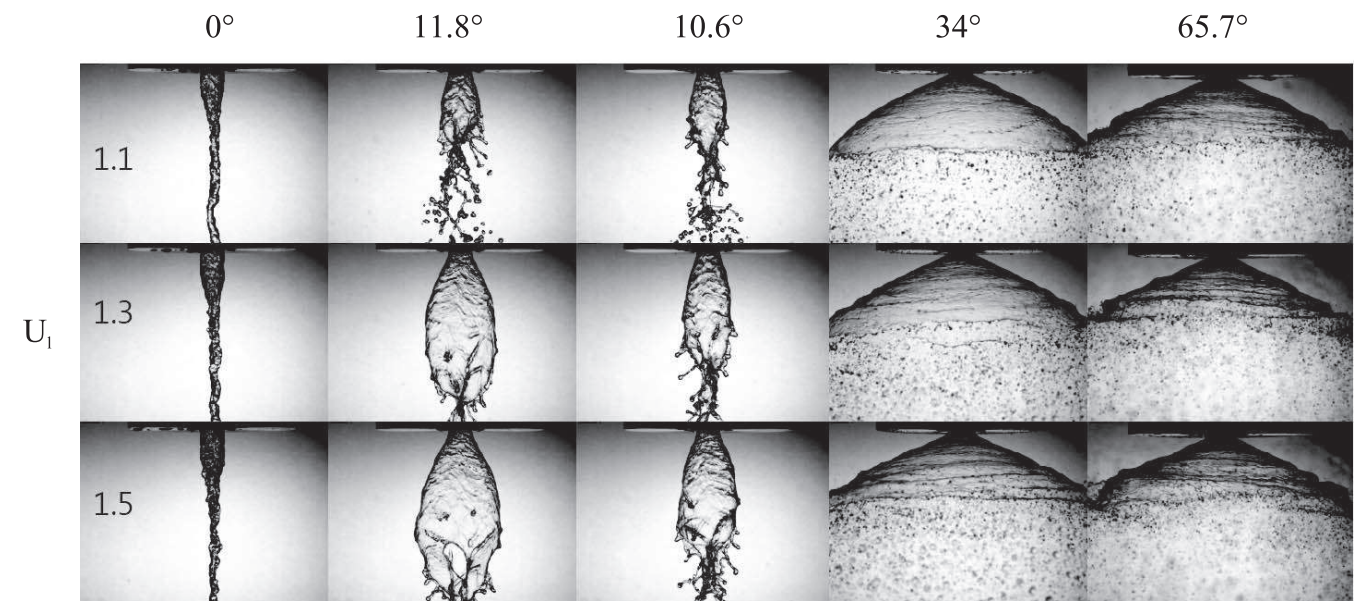

FIG. 2. Estimation of swirl number $S_{w}$ is carried out from measurement of spray angle when $l_{R}=0$. Images are for $H_{l}=$ $0.7 \mathrm{~mm}$ injector. Spray angle is constant when liquid velocity is varied.

gas flow rates are less than $1 \mathrm{l} / \mathrm{h}$ and $10 \mathrm{Nl} / \mathrm{min}$, respectively. Mean liquid and gas velocities derived from respective flow rates are used to compute the dynamic pressure ratio $M=\rho_{g} U_{g}^{2} / \rho_{l} U_{l}^{2}$.

Liquid swirl strength is varied by changing the inlet angle $\alpha$ of the liquid entry holes, as shown in Figure 1. The swirl number $S_{w}$ is defined here as the ratio of the azimuthal velocity $W_{l}$ to the axial velocity $U_{l}$, when there is no recess: $S_{w}=W_{l} / U_{l}$. In order to measure $S_{w}$, a second series of injectors was built, identical to the injectors described above (two $H_{l}$ values and five $\alpha$ values) but with $l_{R}=0 \mathrm{~mm}$. Ratio $S_{w}$ was measured for each geometry (namely, each couple $H_{l}$ and $\alpha$ ) by measuring the spray angle $\gamma$ via direct visualization on these injectors without recess. The angle $\gamma$ is defined here as the sum of both local angles to the vertical measured on the left and on the right: basically $\gamma$ corresponds to the double of the averaged local slope. We then take $S_{w}=\tan \gamma / 2$. Figure 2 shows that $\gamma$ (and therefore $S_{w}$ ) increases with $\alpha$, but depends very little on liquid axial velocity. Measured spray angles and corresponding swirl numbers are indicated in Table I for each geometry. There is an uncertainty of about $5^{\circ}$ for larger spray half-angles, which results in an uncertainty of about $20 \%$ for larger $S_{w}$. In the following, only injectors with $l_{R}=11.4 \mathrm{~mm}$ will be used, and the swirl number at liquid outlet will be estimated via the values of Table I.

Internal flow visualization with a LIF (Laser Induced Fluorescence) method was conducted to investigate the overall form and the interface corrugation of the liquid flow at various experimental conditions. A Coherent Innova 70 Argon laser and a rod lens are used to produce a laser sheet which is set to pass along the central axis of the injector. Images are captured with a Phantom high speed digital camera. Fluorescein is mixed with the liquid (water), and visualization of fluoresced light is carried out through an optical filter, in order to remove any reflected or refracted light. The exposure time and the image number for each experimental condition studied here are $99 \mu \mathrm{s}$ (capture frequency of 10000 frames/s) and 7000 images, respectively.

A digital image processing algorithm was developed with MATLAB to identify the intact liquid area inside the injectors. This method, which relies on appropriate thresholding of raw images, is described in Appendix A.

TABLE I. Values of $S_{w}$ for the injectors used in the present study.

\begin{tabular}{llcccc}
\hline \hline$\alpha(\operatorname{deg})$ & 0 & 10.6 & 11.8 & 34 & 65.7 \\
Measured spray angle (deg) & 0 & 13 & 22 & 113 & 131 \\
$S_{w}$ for $H_{l}=0.5 \mathrm{~mm}$ & 0 & 0.11 & 0.19 & 1.5 & 2.2 \\
Measured spray angle (deg) & 0 & 30 & 42 & 128 & 132 \\
$S_{w}$ for $H_{l}=0.7 \mathrm{~mm}$ & 0 & 0.27 & 0.38 & 2.05 & 2.25 \\
\hline \hline
\end{tabular}



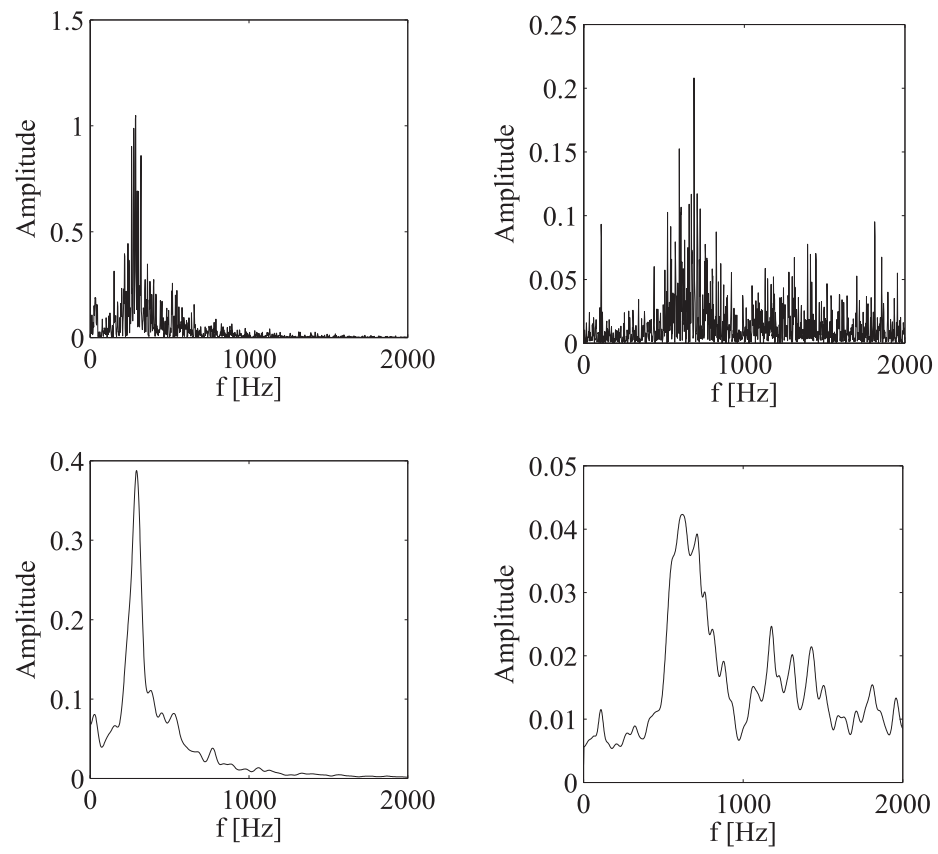

FIG. 3. Left: Examples of spectra of the interface perturbations, for $U_{g}=39 \mathrm{~m} / \mathrm{s}, U_{l}=1 \mathrm{~m} / \mathrm{s}, H_{l}=0.7 \mathrm{~mm}$, and $\alpha=10.6^{\circ}$; top: spectrum of the whole set of images; bottom: the signal is split into 32 segments and averaged; the latter method is preferred when the spectrum is very noisy (see below); right: spectrum obtained for $U_{g}=94 \mathrm{~m} / \mathrm{s}, U_{l}=1 \mathrm{~m} / \mathrm{s}, H_{l}=0.7 \mathrm{~mm}$, and $\alpha=34^{\circ}$ : on the top spectrum two maxima are observed, a broad one $(f=620 \pm 100 \mathrm{~Hz})$ and a very thin one $(f=110 \pm$ $10 \mathrm{~Hz})$.

We also use visualization to study the nature of surface perturbations: for a given downstream distance from liquid injection, we capture the variations of the interface position as a function of time. A spectrum of this quantity is computed via MATLAB. We then observe that for certain conditions a clear maximum frequency dominates the spectrum (see Figure 3 for examples of spectra). This frequency can then be measured as a function of gas and liquid velocities. For a range of conditions two maxima frequencies may be observed (see Figure 3 top right): a lower one whose peak is very narrow, and a larger one whose peak is much broader. The variation of each of these maxima as a function of experimental conditions will be discussed in Sec. III.

Spectra can be carried out for varying downstream positions: Figure 4 shows how spectra vary for two sets of conditions. For $H_{l}=0.7 \mathrm{~mm}, \alpha=11.8^{\circ}, U_{l}=1 \mathrm{~m} / \mathrm{s}$, and $U_{g}=77 \mathrm{~m} / \mathrm{s}$, a constant broad maximum is observed over a relatively long distance downstream. Its frequency remains constant.
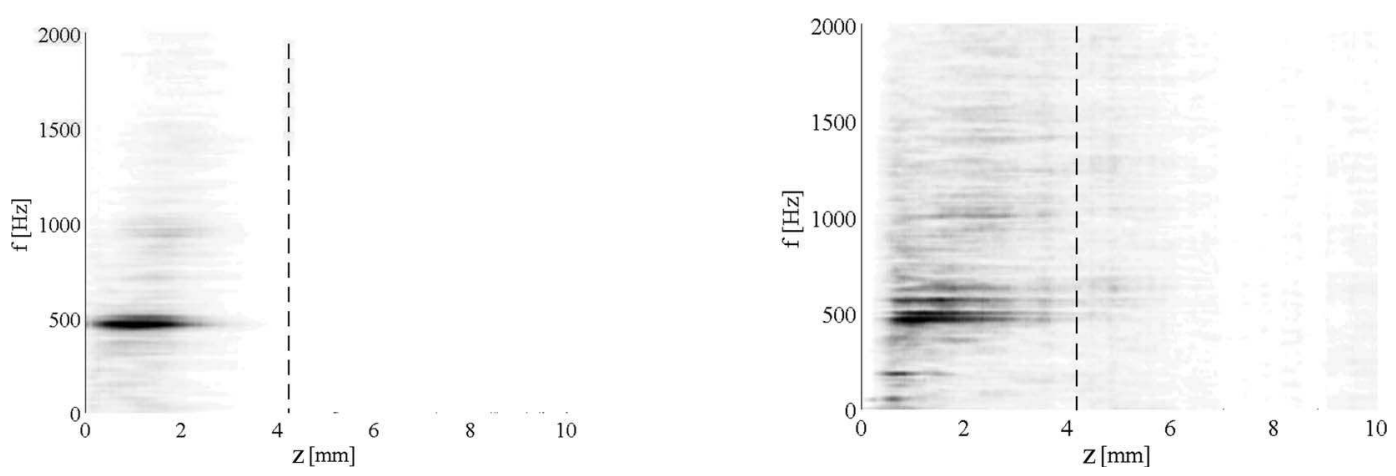

FIG. 4. Spectra for the interface perturbations as a function of downstream distance. The dashed line indicates the end of the liquid tongue. Left: $H_{l}=0.7 \mathrm{~mm}, \alpha=11.8^{\circ}, U_{l}=1 \mathrm{~m} / \mathrm{s}, U_{g}=77 \mathrm{~m} / \mathrm{s}$; Right: $H_{l}=0.7 \mathrm{~mm}, \alpha=34^{\circ}, U_{l}=1 \mathrm{~m} / \mathrm{s}$, $U_{g}=79 \mathrm{~m} / \mathrm{s}$. 
For $H_{l}=0.7 \mathrm{~mm}, \alpha=34^{\circ}, U_{l}=1 \mathrm{~m} / \mathrm{s}$, and $U_{g}=79 \mathrm{~m} / \mathrm{s}$, the additional lower frequency peak of Figure 3 is also observed around $150 \mathrm{~Hz}$, but it disappears over a shorter distance $(z<1 \mathrm{~mm})$. Note also a small peak around $50 \mathrm{~Hz}$ just at the location of injection: this frequency is observed if the spectrum is carried out over a reflection emitted from a solid wall (in spite of the filter some reflections may not be entirely filtered out), but is not observed to survive in the free interface.

\section{EXPERIMENTAL RESULTS}

\section{A. Intact liquid length}

The dynamic pressure ratio $M=\rho_{g} U_{g}^{2} / \rho_{l} U_{l}^{2}$ is usually considered as a main parameter to determine the intact liquid length and the global spray characteristics of GCSC injectors. Figures 5 and 6 show the variation of the internal liquid flow as a function of this parameter at a constant liquid and gas velocity, respectively: the intact liquid length decreases as the dynamic pressure ratio increases. Moreover, the global form of the internal liquid flow remains the same for a constant value of $M$ obtained from different values of liquid and gas velocities: the internal flow visualization for a given value of $M$ is shown in Figure 7. Although the interfacial instability characteristics may be different when changing gas and liquid velocities, it seems that the intact liquid length remains constant for a given value of $M$.

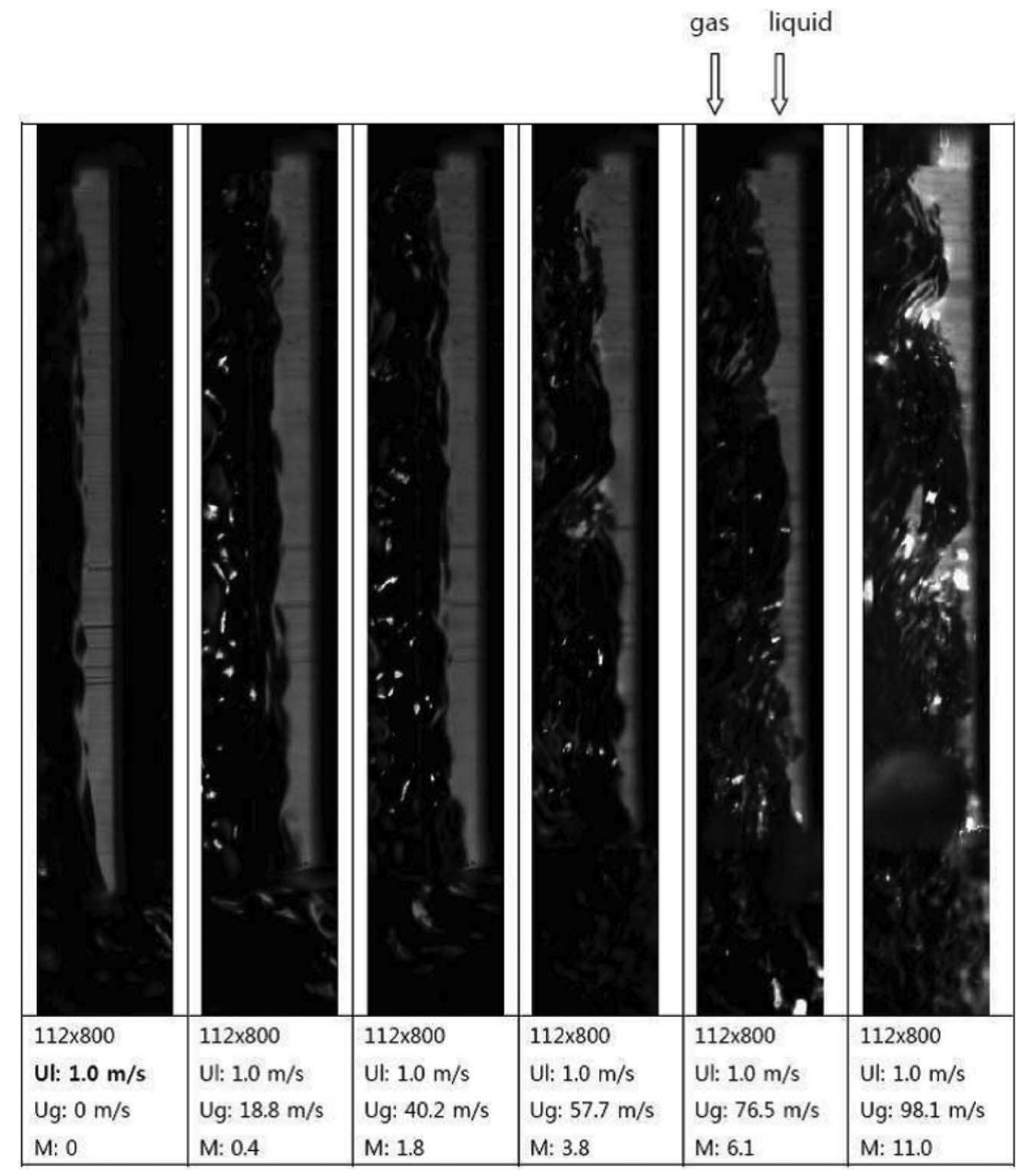

FIG. 5. Variation of the internal liquid flow as a function of the dynamic pressure ratio $M$ at a constant $U_{l}=1.0 \mathrm{~m} / \mathrm{s}\left(H_{l}=\right.$ $0.7 \mathrm{~mm}, \alpha=65.7^{\circ}, M=0 \rightarrow 11.0$ from left to right). 


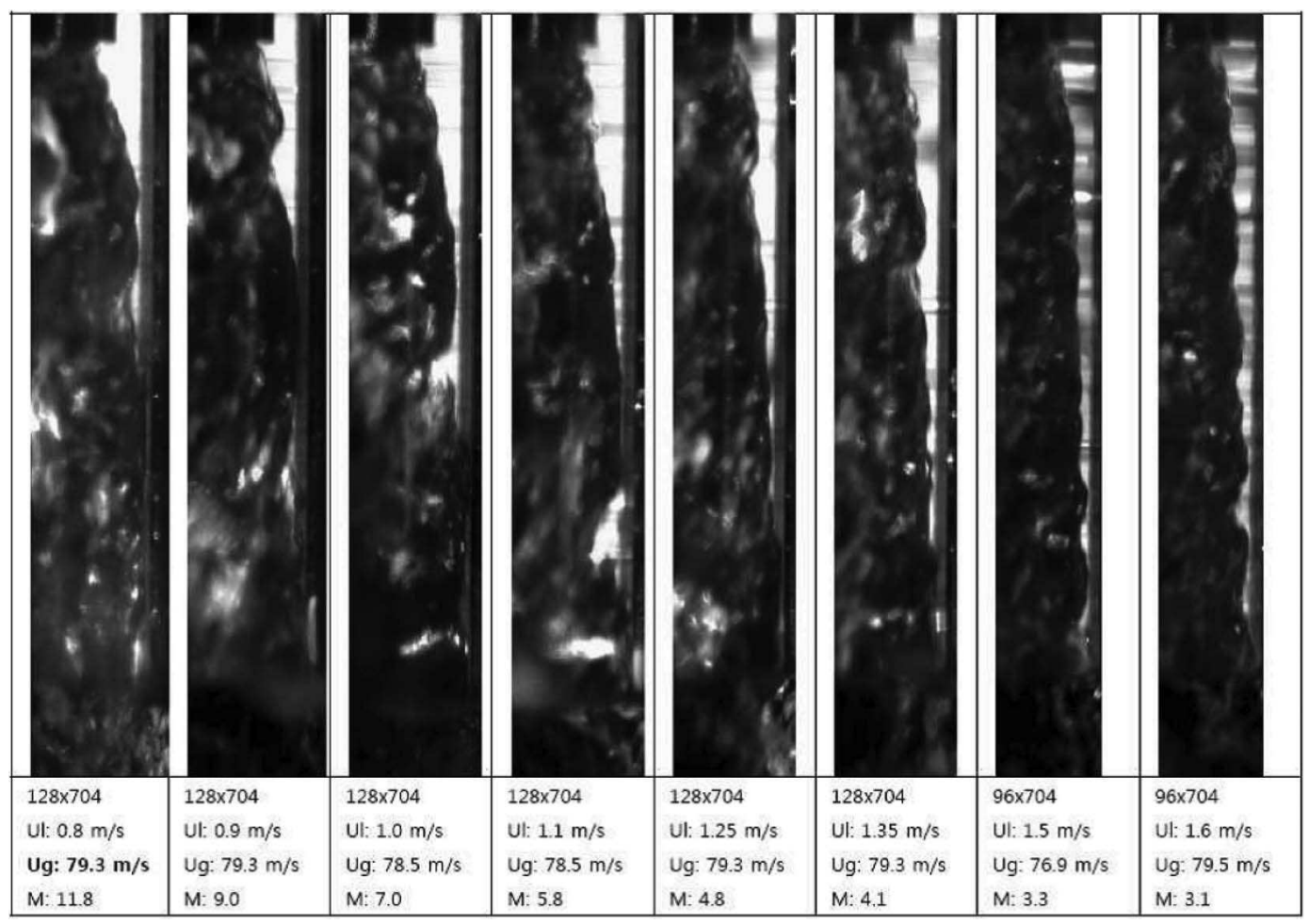

FIG. 6. Variation of the internal liquid flow as a function of the dynamic pressure ratio $M$ at a constant $U_{g}=79 \mathrm{~m} / \mathrm{s}\left(H_{l}=\right.$ $0.7 \mathrm{~mm}, \alpha=65.7^{\circ}, M=11.8 \rightarrow 3.1$ from left to right).

We now turn to the influence of swirl on the liquid intact length. Figure 8 shows the variation of the internal liquid flow at various $S_{w}$ for a same set of axial phasic velocities: the intact liquid length increases when the swirl strength increases. For both constant $M$ of Figure 8, the intact liquid length reaches the end of the injector when swirl is increased up to $S_{w}=2.3\left(\alpha=65.7^{\circ}\right.$ for $\left.H_{l}=0.7 \mathrm{~mm}\right)$.

Figure 9 left shows the effects of the dynamic pressure ratio and swirl strength on $L_{b}$ for injectors with $H_{l}=0.5 \mathrm{~mm}$. As the dynamic pressure ratio increases and the swirl strength decreases, the intact liquid length somewhat decreases. The data of Figure 9 suggest a power law dependency between the intact liquid length and the dynamic pressure ratio. As the swirl velocity increases $L_{b}$ increases: the liquid film becomes more stable, being forced against the injector inner wall with the increment of the azimuthal momentum. Schumaker et al. ${ }^{7}$ suggested to use the total dynamic pressure ratio $M_{t}=\rho_{g} U_{g}^{2} / \rho_{l} U_{l}^{2}\left(1+S_{w}^{2}\right)$ to capture the influence of swirl on the intact liquid length: Figure 9 right shows that this parameter fails to appropriately scale our data, and that dispersion is increased. Finally, it must be noted that the decrease of the intact liquid length as $M^{-1.2}$ observed here is steeper than the decrease in $M^{-1 / 2}$ observed in mixing layer experiments (see, e.g., Eggers and Villermaux ${ }^{9}$ ), and which is attributed to stripping of the liquid by the fast gas stream. We think that in the present case the thickness of the liquid stream is mainly driven by the acceleration of the liquid by the fast gas stream, which leads to thinning of the liquid film. In addition, spray measurements indicate that few droplets are detached from the film within the injector recess zone, and that at any rate these droplets are very small, ${ }^{10}$ so that the mass loss due to stripping is actually quite weak. At large $M$ values, where stripping becomes more efficient, the $M^{-1 / 2}$ dependency may eventually be recovered (see lower slope for $M>2$ on Figure 9). Experiments at higher $M$ are needed to confirm this point.

Figure 10 shows the variations of intact liquid length as a function of $M$ and $S_{w}$, this time for $H_{l}=0.7 \mathrm{~mm}$. The same overall decrease with $M$ and increase with $S_{w}$ is observed. The slopes of Figure 9 are reported on Figure 10 to facilitate a comparison: the power law behavior is not clearly 


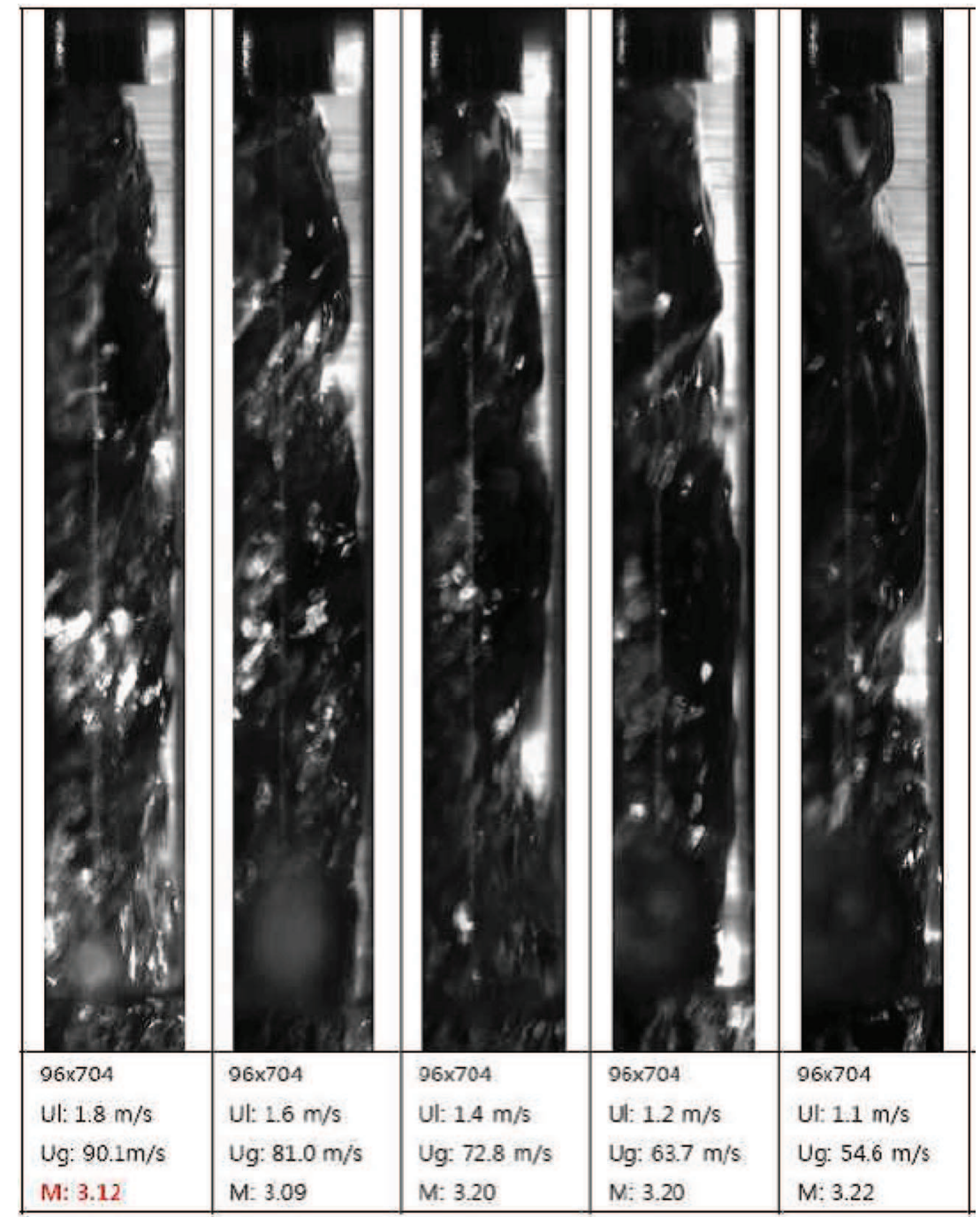

FIG. 7. Variation of the internal liquid flow at a constant $M=3.1$ obtained from different combinations of liquid/gas velocities $\left(H_{l}=0.5 \mathrm{~mm}, \alpha=65.7^{\circ}, U_{l}=1.1 \rightarrow 1.8 \mathrm{~m} / \mathrm{s}, U_{g}=54.6 \rightarrow 90.1 \mathrm{~m} / \mathrm{s}\right)$.

seen. Exponent -1.2 (dashed line) may be valid for $M<4$, but data for larger $M$ clearly show a much weaker impact of $M$, which is overestimated by the $M^{-0.5}$ trend (dotted line).

\section{B. Interfacial instability}

Figures 11 and 12 show the variations of the most unstable frequency in surface perturbations, as a function of gas velocity. The error bars are related to the uncertainty on localizing the maximum in the spectrum, typically the width of the maximum peak in the spectrum. All in all the main trend is that frequency increases as a function of gas velocity, for all injector conditions (i.e., for all initial swirl in the system). This is consistent with what is known for shear instabilities in two-phase mixing layers, where the frequency of the instability is mostly controlled by velocity of the fast gas stream. ${ }^{9}$ However, a peculiar feature of the instability in the present study can be seen in Figure 12(b), where two frequencies seem to coexist: in addition to the frequency increasing with $U_{g}$, there is a constant frequency, comprised between $150 \mathrm{~Hz}$ and $200 \mathrm{~Hz}$ for the large range of gas velocities investigated. While the spectrum associated with the larger frequency is usually broad (width larger than $100 \mathrm{~Hz}$ ), the maximum associated with the lower frequency independent of $U_{g}$ is very narrow (width smaller 

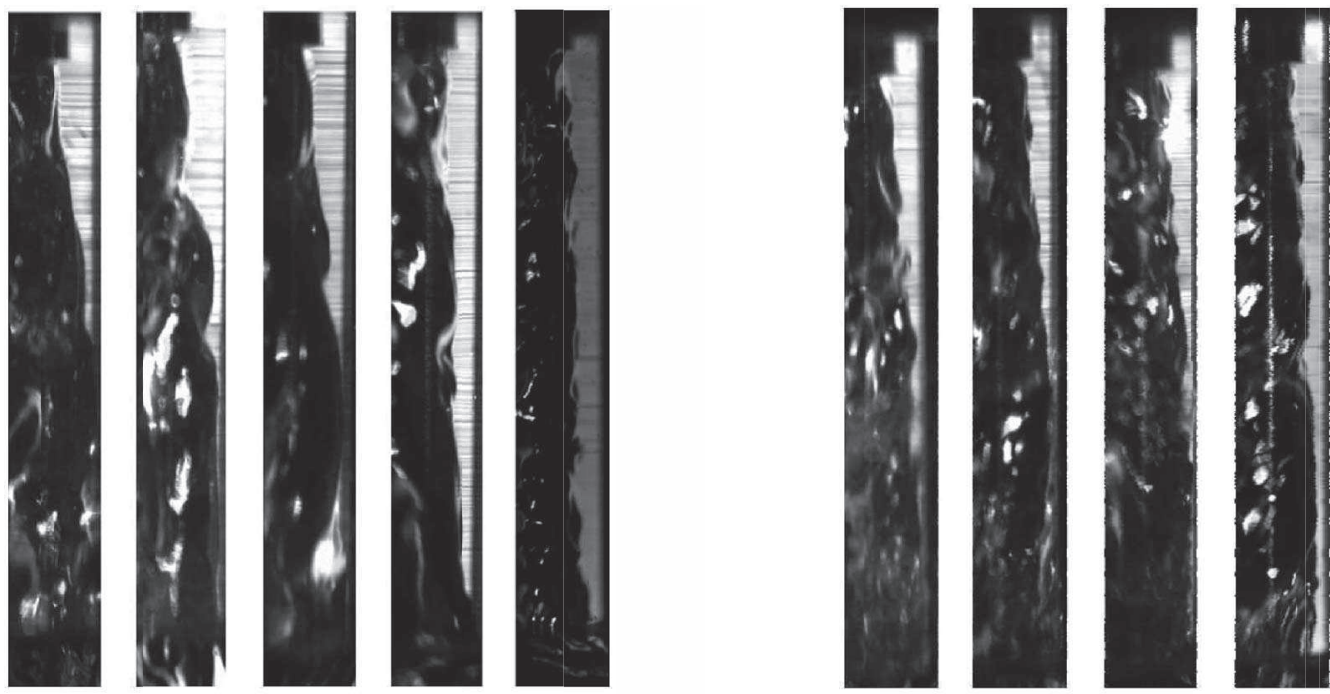

$$
\mathrm{S}_{\mathrm{W}}=0 \quad \mathrm{~S}_{\mathrm{W}}=0.27 \quad \mathrm{~S}_{\mathrm{W}}=0.38 \quad \mathrm{~S}_{\mathrm{W}}=2.0 \quad \mathrm{~S}_{\mathrm{W}}=2.3
$$

$\mathrm{S}_{\mathrm{W}}=0$

$\mathrm{S}_{\mathrm{W}}=0.19$

$\mathrm{S}_{\mathrm{W}}=1.5$

$\mathrm{S}_{\mathrm{W}}=2.2$

FIG. 8. Variation of the internal liquid flow as a function of swirl strength for: Left $M=1.8, U_{l}=1.0 \mathrm{~m} / \mathrm{s}, U_{g}=39.2 \mathrm{~m} / \mathrm{s}$, and $H_{l}=0.7 \mathrm{~mm}$; Right: $M=1.46, U_{l}=1.4 \mathrm{~m} / \mathrm{s}, U_{g}=49.1 \mathrm{~m} / \mathrm{s}, H_{l}=0.5 \mathrm{~mm}$.

than $5 \mathrm{~Hz}$ ). Figure 3 shows an example of a spectrum where both frequencies coexist. Figure 13 left shows the superposition of the data for $U_{l}=1 \mathrm{~m} / \mathrm{s}, H_{l}=0.7 \mathrm{~mm}$, and all $S_{w}$ : surprisingly it can be seen that frequency does not vary much when the swirl is varied. Figure 13 right shows that the superposition is even better when data are sorted as a function of the width of the spectrum. The frequency of data points corresponding to a wide spectrum increases steadily with $U_{g}$ independent of $S_{w}$, and the frequency of data points corresponding to a narrow spectrum is approximately constant. There is however an exception: the single data point corresponding to $S_{w}=0$ (cross) in Figure 13(c), for $U_{g}=60 \mathrm{~m} / \mathrm{s}$, has a very sharp spectrum (width at mid height of the order of $2 \mathrm{~Hz}$ ), but a much larger frequency than other data points with a similar spectrum.

It is known ${ }^{11-13}$ that the inviscid instability of a two-phase planar shear layer is controlled by the thickness of the gas vorticity layer $\delta_{g}$ : more precisely, the wavelength $\lambda$ can be shown to be directly proportional to $\delta_{g}$, with $\lambda \sim \delta_{g} / \sqrt{r_{\rho}}$ where $r_{\rho}$ is the density ratio $r_{\rho}=\rho_{g} / \rho_{l}$. The frequency of the
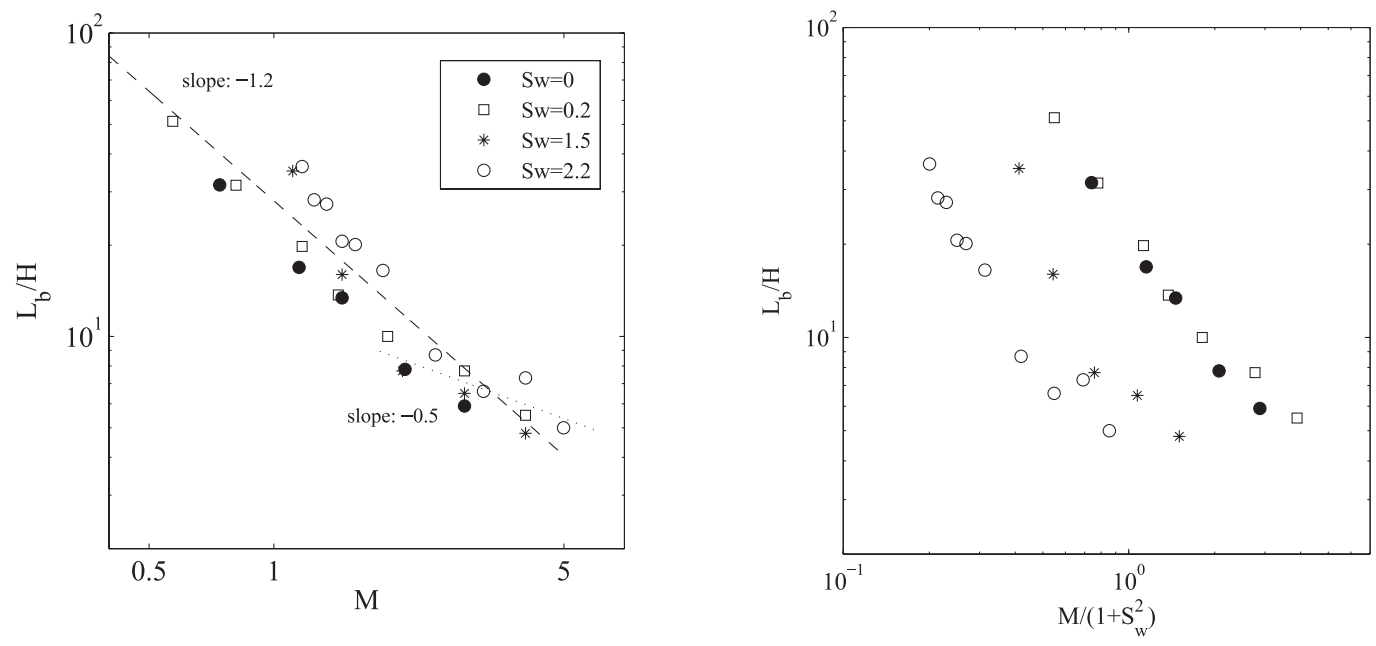

FIG. 9. Left: Variation of the intact liquid length as a function of the momentum flux ratio $M$, for varying swirl strengths $\left(H_{l}\right.$ $\left.=0.5 \mathrm{~mm}, d_{g}=10.8 \mathrm{~mm}\right)$. Right: same data, plotted as a function $M /\left(1+S_{w}^{2}\right)$. 


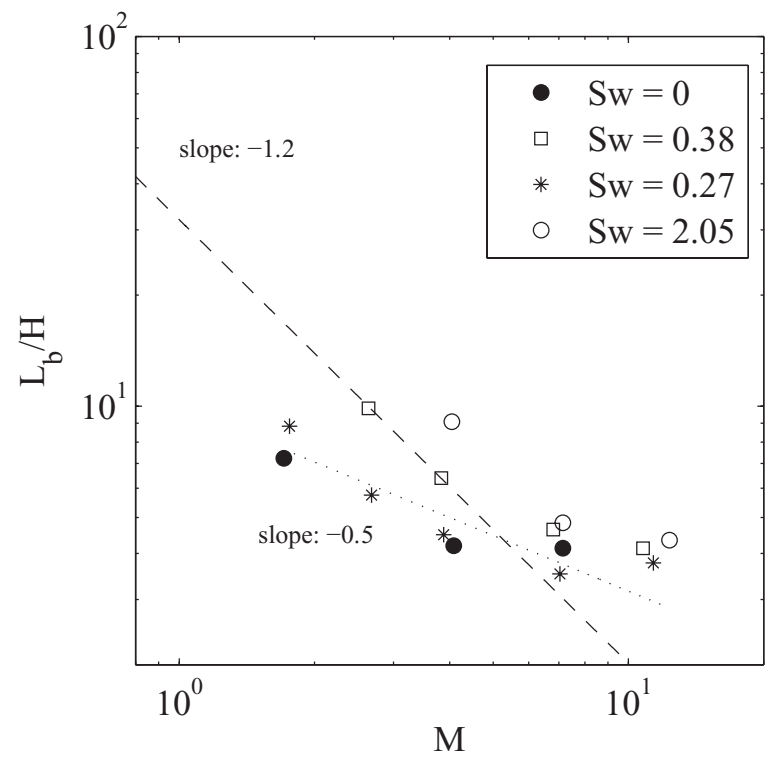

FIG. 10. Variation of the intact liquid length as a function of the momentum flux ratio $M$, for varying swirl strengths $\left(H_{l}=\right.$ $0.7 \mathrm{~mm}, d_{g}=10.8 \mathrm{~mm}$ ).

instability can then be estimated from the ratio of the wave velocity $U_{c}$ to $\lambda$ : wave velocity is the velocity of the frame in which dynamic pressure in both phases are balanced, ${ }^{14} U_{c} \approx \sqrt{r_{\rho}} U_{g}$. This leads to a frequency scaling as $f \sim r_{\rho} U_{g} / \delta_{g}$. This estimate is valid when the dynamic pressure ratio $M$ is large, which may not be the case for the lower $U_{g}$ investigated here. Nonetheless, comparison can be attempted between this prediction and our experimental data: gas vorticity thickness $\delta_{g}$ has been determined via hotwire measurements (with a single sensor normal probe, $1.25 \mathrm{~mm}$ long and $0.5 \mu \mathrm{m}$ diameter, 55P11 of DANTEC Dynamics), and was found to follow $\delta_{g} \approx 0.18 d_{g} R e_{g}^{-1 / 7}$ for the conditions of our experiment. We have noted $R e_{g}=U_{g} R / v_{g}$ the Reynolds number in the gas stream, with $v_{g}$ the gas kinematic viscosity. This would yield frequencies in the range [1-50] Hz according to the above prediction for $f$, quite below the frequency observed experimentally. Inviscid predictions have been observed to underestimate the value of frequencies observed experimentally by a factor 2 or 3 in particular in the round jet configuration, ${ }^{12}$ but here the factor 10 between
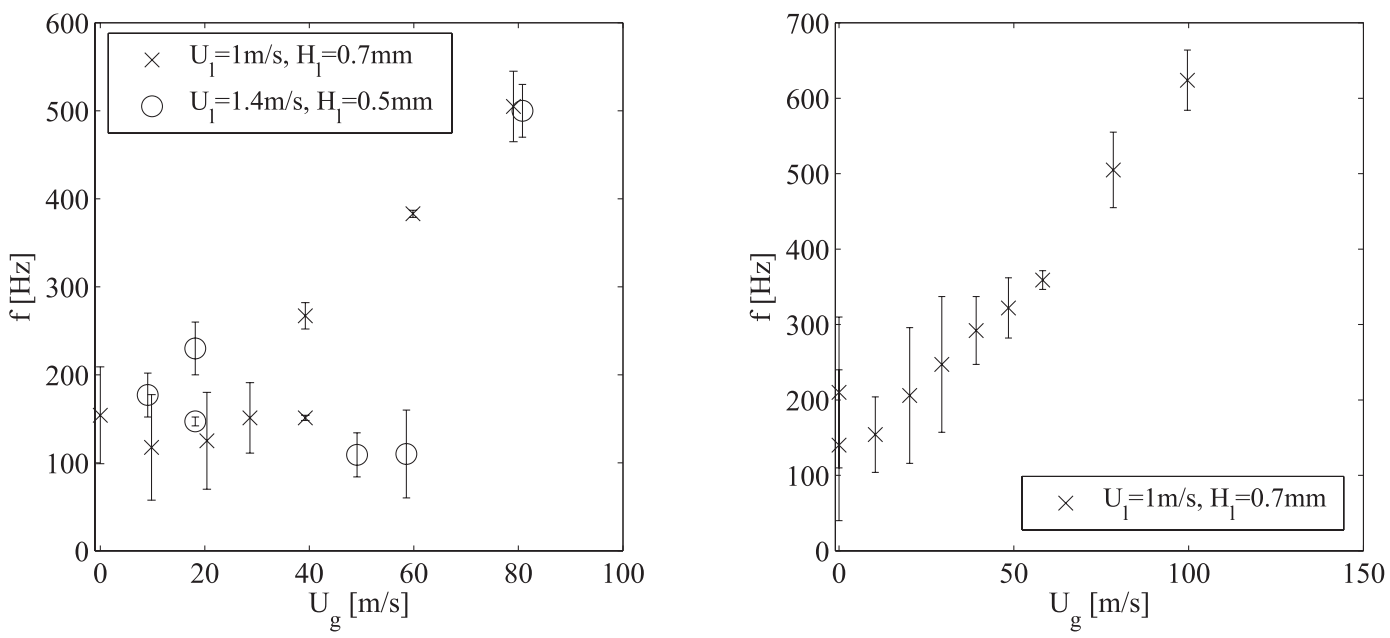

FIG. 11. Variation of the frequency of the surface instability as a function of gas velocity; Left: $S_{w}=0$; Right: $S_{w}=0.11$. 


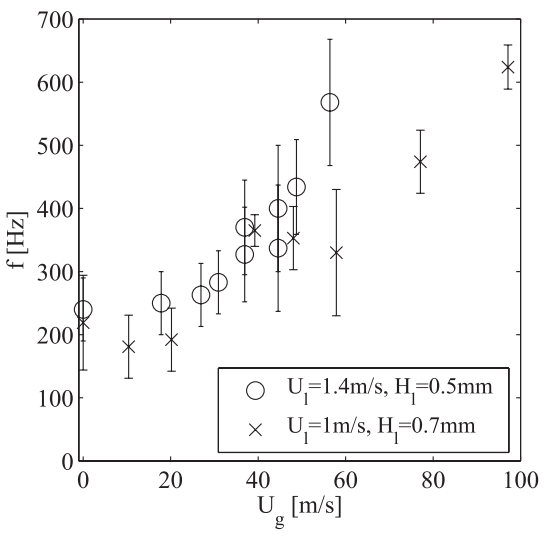

(a)

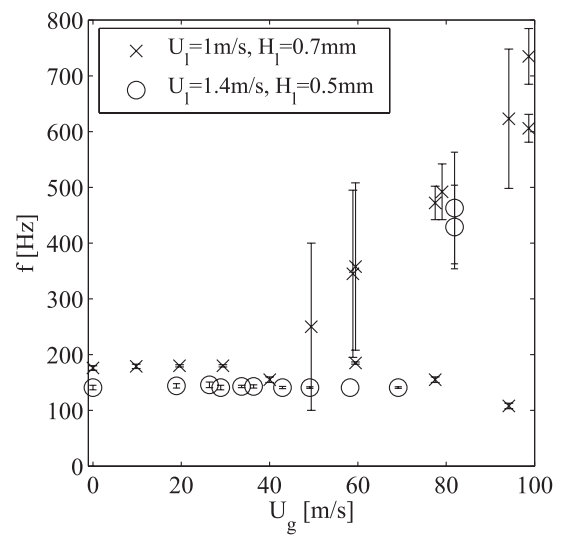

(b)

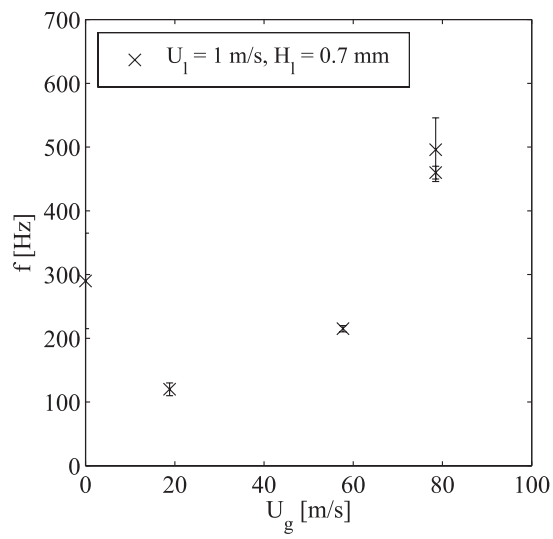

(c)

FIG. 12. Variation of the frequency of the surface instability as a function of gas velocity; (a) $S_{w}=0.2$; (b) $S_{w}=1.5$; (c) $S_{w}=2.2$.

experimental value and estimate suggest that a different mechanism may be at play. We will discuss this in subsequent sections when turning to stability analysis.

Figure 14 shows the amplitude of the perturbation as a function of downstream distance, for two swirl numbers $\left(S_{w}=0\right.$ and $S_{w}=0.11$ ), four different gas velocities, and fixed $U_{l}=1 \mathrm{~m} / \mathrm{s}$ and $H_{l}=0.7 \mathrm{~mm}$. Amplitude is here defined as the $\mathrm{rms}$ value of the interface position in pixels. Amplitude for $U_{g}=0 \mathrm{~m} / \mathrm{s}$ and $U_{g}=20 \mathrm{~m} / \mathrm{s}$ are relatively close, indicating that gas velocity only has a significant effect on the surface instability for $U_{g}>40 \mathrm{~m} / \mathrm{s}$ : this is consistent with the results of Figure 13, which show that frequency of the instability converges to a finite value when gas velocity tends to zero, and that frequency departs from this limit value for $U_{g}$ between 20 and $40 \mathrm{~m} / \mathrm{s}$. For all $U_{g}>$ $20 \mathrm{~m} / \mathrm{s}$, the amplitude first increases, then reaches a maximum and eventually decreases: interface destabilization is mainly effective at short distances from the liquid outlet. For example, for $U_{g}=$ $78 \mathrm{~m} / \mathrm{s}$ and both $S_{w}$, the maximum is found just before $2 \mathrm{~mm}$ from injection. At larger downstream distances wave amplitude decreases: as mentioned earlier stripping of droplets is negligible within the recess zone, and we attribute this stabilization of the liquid interface to the acceleration, and simultaneous thinning, of the liquid film by the gas stream.

We also note on Figure 14 that until the maximum is reached growth of the waves is mostly linear (algebraic): a very short convex region may be observed close to injection, but it is not long enough to allow for a proper spatial growth rate measurement. In addition, to be valid growth rate measurement cannot be made on raw amplitude but would have to take into account a precise calibration along the radius of the injector, since refraction effects across the injector are expected to stretch distances at larger radii. 


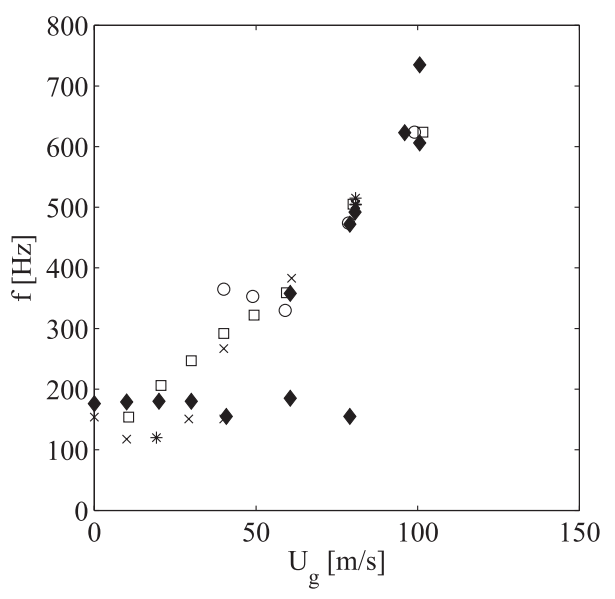

(a)

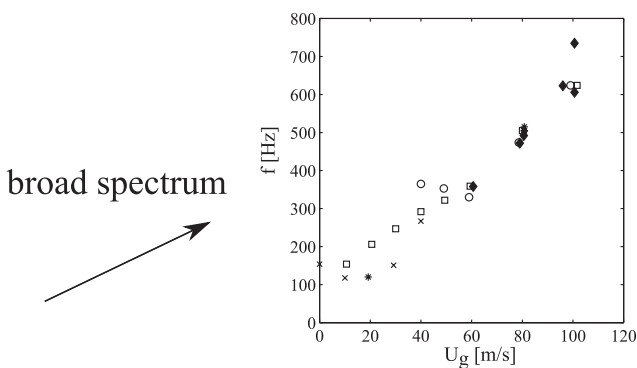

(b)
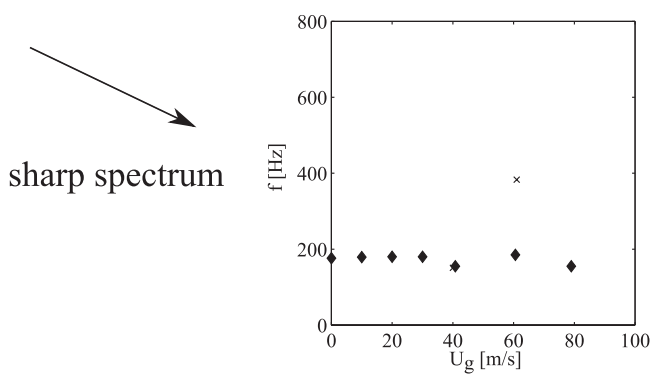

(c)

FIG. 13. Variation of the frequency of the surface instability as a function of gas velocity. (a) Superposition of frequencies for $U_{l}=1 \mathrm{~m} / \mathrm{s}, H_{l}=0.7 \mathrm{~mm}$ and all $S_{w}$ investigated: $\times S_{w}=0 ; \square S_{w}=0.11 ; \circ S_{w}=0.2 \nleftarrow$; $S_{w}=1.5 ; * S_{w}=2.2$. (b) Data for which the maximum frequency corresponds to a broad peak; (c) data for which the maximum frequency corresponds to a sharp peak in the spectrum.

\section{MODEL FOR THE LIQUID INTACT LENGTH}

In order to understand the impact of swirl and of gas velocity in the data of Figures 9 and 10, we now present a simple model based on energy conservation. We write Bernoulli's law in a frame rotating at angular velocity $W_{l} / R$, where $W_{l}$ is the fluid azimuthal velocity. In this frame, the fluid is subject to a centrifugal force deriving from potential $E_{p}=-\frac{1}{2} \rho_{l} W_{l}^{2}$. We consider two points lying on the same streamline at the surface of the liquid, one located at the fluid outlet (axial
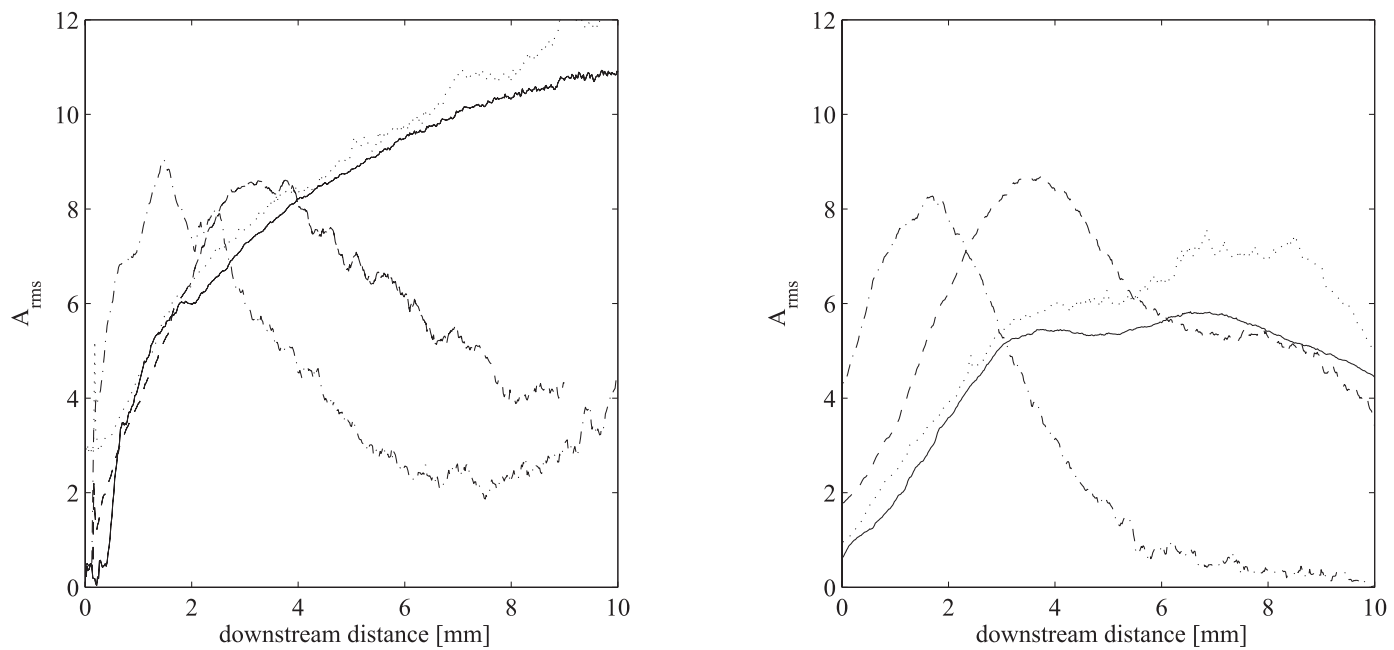

FIG. 14. Amplitude of the surface perturbation (rms value of amplitude in pixels) as a function of downstream distance for $S_{w}=0$ (left) and $S_{w}=0.27$ (right), fixed $U_{l}=1 \mathrm{~m} / \mathrm{s}$ and $H_{l}=0.7 \mathrm{~mm}$ : solid line $U_{g}=0 \mathrm{~m} / \mathrm{s} ;$ dotted line $U_{g}=20 \mathrm{~m} / \mathrm{s}$; dashed line $U_{g}=40 \mathrm{~m} / \mathrm{s}$; dashed-dotted line $U_{g}=78 \mathrm{~m} / \mathrm{s}$. 
velocity $U_{1}$, swirl velocity $W_{1}$ ), and the other one located at a downstream distance $z$ (axial velocity $U_{2}$, swirl velocity $W_{2}$ ). Assuming steady motion and neglecting gravity and viscous effects, energy conservation writes

$$
\frac{1}{2} \rho_{l}\left(U_{2}^{2}-U_{1}^{2}\right)-\frac{1}{2} \rho_{l}\left(W_{2}^{2}-W_{1}^{2}\right)=E_{\text {drag }},
$$

where $E_{d r a g}$ is the energy provided to the liquid by the fast central gas stream between points 1 and 2. This term can be estimated as

$$
E_{\text {drag }} \approx K \rho_{g} U_{g}^{2}(2 \pi R z) z /\left(2 \pi R z\left(h_{1}+h_{2}\right) / 2\right)=2 K \rho_{g} U_{g}^{2} \frac{z}{h_{1}+h_{2}},
$$

where $h_{1}$ (resp. $h_{2}$ ) is the liquid thickness at point 1 (resp. 2) and coefficient $K$ is a drag coefficient. This coefficient is expected to mainly depend on interface corrugation. The expression for $E_{\text {drag }}$, work of the drag force per liquid unit volume, is derived by assuming $E_{\text {drag }}=$ dragforce $\times$ length/volume. Surface $2 \pi R z$ and volume $\pi R z\left(h_{1}+h_{2}\right)$ are expressed to lowest order in $h / R$. Assuming stripping is negligible mass conservation yields $U_{1} h_{1}=U_{2} h_{2}$. Angular momentum conservation gives

$$
W_{1}\left(R-h_{1}\right)=W_{2}\left(R-h_{2}\right) .
$$

Writing again that thicknesses $h_{1}$ and $h_{2}$ are small compared to $R$ (largely true in our experimental case) leads to

$$
W_{2} \approx W_{1}\left(1-\frac{h_{1}-h_{2}}{R}\right) .
$$

We report this in energy conservation, and obtain (at lower order in $h / R$ )

$$
\frac{1}{2} \rho_{l} U_{1}^{2}\left(\frac{h_{1}^{2}}{h_{2}^{2}}-1\right)+\rho_{l} \frac{W_{1}^{2}}{R}\left(h_{1}-h_{2}\right)=2 K \rho_{g} U_{g}^{2} \frac{z}{h_{1}+h_{2}} .
$$

Solving this equation for $h_{2}(z)$ would give the liquid thickness as a function of downstream distance $z$. Here, we just write that the liquid intact length $L_{b}$ is defined by: $\left.\frac{d h_{2}}{d z}\right|_{z=0}=-\frac{h_{1}}{L_{b}}$. Derivating Eq. (1), and considering $h_{1}=H_{l}$ (initial liquid height) then gives $L_{b}$

$$
\frac{L_{b}}{H_{l}}=\frac{1}{K}\left(\frac{1}{M}+\frac{S_{w}^{2}}{M} \frac{H_{l}}{R}\right) \text {. }
$$

We plot on Figure 15 the data of Figures 9 and 10, but this time as a function of $M /\left(1+S_{w}^{2} H_{l} / R\right)$, the parameter suggested by Eq. (2): the data for larger $S_{w}$ (symbol $\circ$ on Figures 9 and 10, and symbols $\circ$ and $\downarrow$ on Figure 15) are indeed collapsed on the main trend. We call the attention of the reader to the fact that data of Figures 9 and 10 were for $L_{b} / H$, while our model is for $L_{b} / H_{l}$ : we justify the comparison by our observation that the experimental liquid height $H$ does not vary significantly for our whole set of measurements, and that the ratio $H / H_{l}$ is therefore approximately constant. Figure 15 next shows the superposition of the data for both $H_{l}$, and their comparison to the predicted slope: The dashed line shows the slope of -1 suggested by Eq. (2). This slope is consistent with experimental data. As mentioned in Sec. III the trend for $M>4$ seems to be different, with a lower slope: this may be due to non-negligible stripping of the liquid by the fast gas stream for these large dynamic pressure ratio conditions. Another possible explanation could be that drag coefficient $K$ may itself depend on gas velocity: as mentioned in Sec. III the average wave amplitude over the whole recess length is globally lower at large gas velocities (see Figure 14). A reduction in interface corrugation may then induce a lower $K$ (and hence a larger $L_{b}$ ) for these conditions. The drag coefficient $K$ estimated from the dashed line in Figure 15 is $K \approx 0.045$ : this is a correct order of magnitude for friction drag over a rough surface and pressure drag due to surface corrugations.

\section{INVISCID LINEAR STABILITY ANALYSIS}

\section{A. Method}

In order to investigate how swirl in the base flow may affect the inviscid mechanism discussed in Sec. III B, we carry out an inviscid linear stability analysis where swirl is taken into account. We 


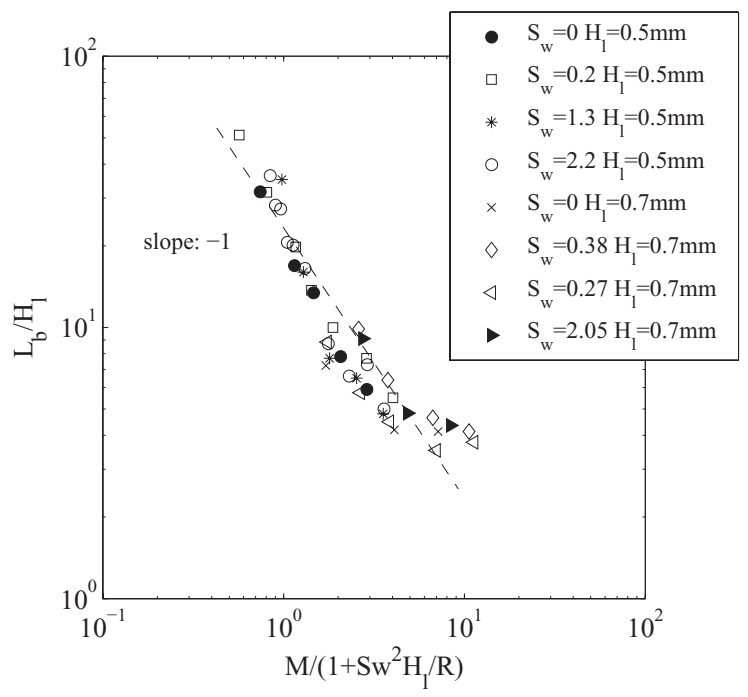

FIG. 15. Liquid intact length as a function of $M /\left(1+S_{w}^{2} H_{l} / R\right)$ for both $H_{l}$ investigated, and comparison to predicted slope. The data of Figures 9 and 10 for larger $S_{w}$ are collapsed on the main trend.

write down Navier Stokes equations + mass conservation in axisymmetric coordinates $(z, r, \theta)$, and write that velocity is the superposition of a base flow $\mathbf{U}=(U(r), 0, W(r))$ and of a small perturbation $\mathbf{u}=(u, v, w)$. Component $U$ is therefore the base flow axial velocity, and component $W$ the base flow azimuthal velocity. We then linearize the system and look for normal solutions of the form $\tilde{\mathbf{u}}(k, r, n, \omega) e^{i(k z+n \theta+\omega t)}$. Away from the interface we can solve for the radial velocity perturbation $\tilde{v}$, and find the following differential equation:

$$
\begin{aligned}
\frac{d^{2} \tilde{v}}{d r^{2}}+\frac{d \tilde{v}}{d r} & {\left[\frac{1}{r}+\frac{2 n^{2}}{r^{3}} \frac{1}{k^{2}+\frac{n^{2}}{r^{2}}}\right]-\tilde{v}\left[\frac{1}{r^{2}}+\left(k^{2}+\frac{n^{2}}{r^{2}}\right)\left[1+\frac{2 W}{A^{2} r}\left(\frac{W}{r}+\frac{d W}{d r}\right)\right]+\right.} \\
& \left.\frac{1}{A}\left(\frac{d B}{d r}-\frac{1}{r} \frac{d A}{d r}-\frac{2 i n W}{r^{3}}+\frac{2 i n W}{r^{2}} \frac{B}{A}\right)+\frac{1}{k^{2}+\frac{n^{2}}{r^{2}}} \frac{2 n^{2}}{r^{3}}\left(\frac{B}{A}-\frac{1}{r}\right)\right]=0,
\end{aligned}
$$

where we have introduced coefficients

$$
\begin{aligned}
& A=i\left(-\omega+k U+\frac{n}{r} W\right), \\
& B=i\left[k \frac{d U}{d r}+\frac{n}{r}\left(\frac{W}{r}+\frac{d W}{d r}\right)\right] .
\end{aligned}
$$

In the inner region of the injector, and away from the liquid interface, the base gas flow is such that $W=0$, and the axial velocity is constant: We note $r_{0}$ the radial distance below which we assume $W=0$ and $U$ constant. It can be shown that in this region pressure is solution of a modified Bessel equation, and the radial velocity can therefore be directly obtained in terms of derivatives of modified Bessel functions of the first kind $I_{n}(r): \tilde{v}=\frac{d I_{n}(r)}{d r}=I_{n}^{\prime}(r)$.

We therefore integrate three independent solutions of Eq. (3): one solution from the solid wall to the interface in the liquid phase, and two other solutions from an arbitrary position $r_{0}$ (supposedly in the constant gas velocity region) to the interface in the gas phase. Position $r_{0}$ is taken such that $r_{0}=d_{g} / 2-5 \delta_{g}$. Orthonormalization is enforced within the couple of solutions during integration to ensure both solutions remain independent (see, for example, Asmolov ${ }^{15}$ for a description of this procedure in a similar context). Integration is carried out with a Dormand-Prince Runge-Kutta method in Fortran. We finally impose continuity of normal velocity $\tilde{v}$ and continuity of normal stress (pressure) across $r_{0}$, and across the interface where surface tension $\sigma$ is taken into account. These four conditions enforced on the three integrated solutions and on $I_{n}^{\prime}(r)$ yield the dispersion relation. We chose to solve for temporal modes, i.e., real $k$ and complex $\omega$. 

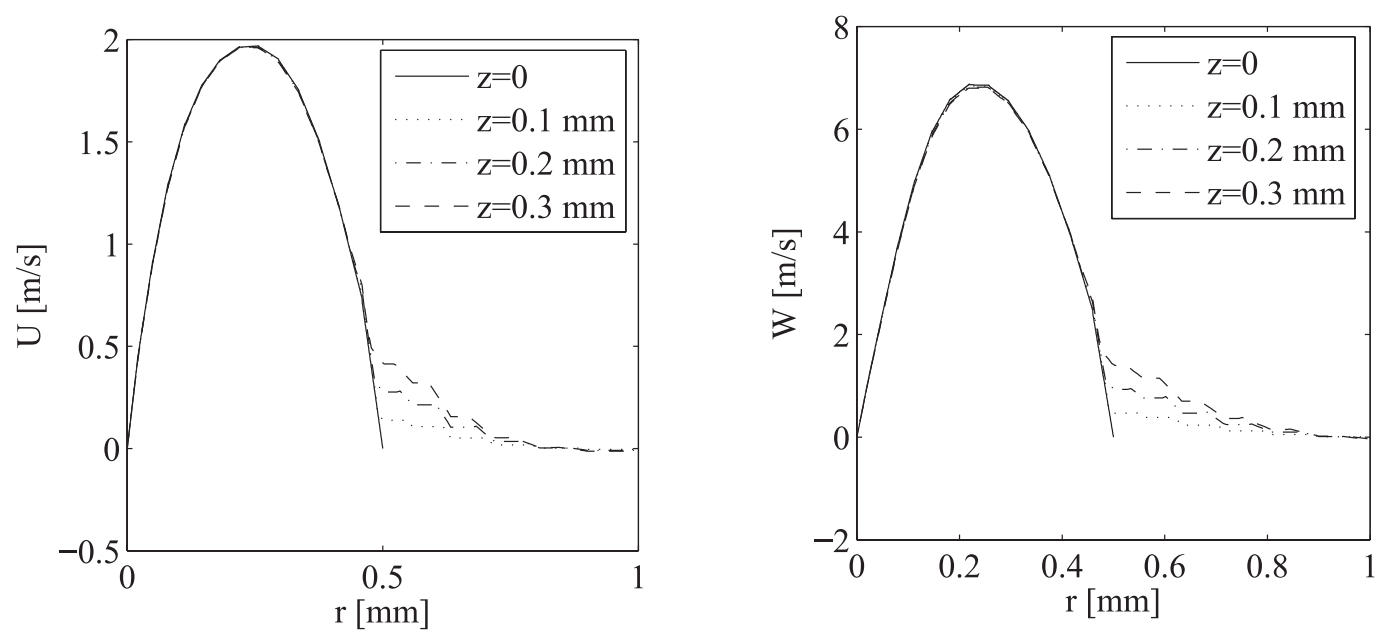

FIG. 16. Base flow profiles obtained via numerical simulation, and injected in the stability analysis. Left: axial velocity; right: orthoradial velocity; $\alpha=65.7^{\circ}$ and $H_{l}=0.5 \mathrm{~mm}, U_{l}=1.4 \mathrm{~m} / \mathrm{s}$. The origin of distance $r$ is taken at the outer wall. Profiles are shown for four different downstream distances $z$.

The base flow in the gas phase is modelled with error functions with a vorticity thickness $\delta_{g}$ equal to the one measured experimentally (see Sec. II)

$$
U(r)=U_{i}+\left(U_{g}-U_{i}\right) \operatorname{erf}\left(\frac{R-H_{l}-r}{1.13 \delta_{g}}\right) \text { and } W(r)=W_{i}\left(1-\operatorname{erf}\left(\frac{R-H_{l}-r}{1.13 \delta_{g}}\right)\right) .
$$

The liquid base flow at the end of the annular channel is estimated via a volume of fluid (VOF) numerical simulation of air-water two-phase flow with Fluent. The simulation is carried out without the central fast gas jet. Profiles of Figure 16 are obtained for the $\alpha=65.7^{\circ}$ and $H_{l}=0.5 \mathrm{~mm}$ injector, for $U_{l}=1.4 \mathrm{~m} / \mathrm{s}$, at the downstream location where the liquid meets the fast gas stream in the experiment. They show a well developed axial velocity profile (liquid Reynolds number based on axial velocity is $R e_{l}=700$ ), with liquid boundary layers of the order of the channel half-height. The profiles are almost parabolic. We could check that in the simulation these profiles are independent of the azimuthal position (not shown here). This simulation predicts a ratio $W_{l} / U_{l} \approx 3.5$ at the end of the liquid channel for this injector $\left(U_{\max } \approx 2 \mathrm{~m} / \mathrm{s}\right.$ and $W_{\max } \approx 7 \mathrm{~m} / \mathrm{s}$ in Figure 16). This ratio is larger than the ratio $S_{w}=2.2$ observed experimentally for the same injector without any recess, see Sec. II: however, these values of $S_{w}=2.2$ and $S_{w}=3.5$ actually correspond to relatively close spray half angles (namely $68^{\circ}$ and $74^{\circ}$ ), meaning that the simulated swirl is actually not so different from the observed one. Based on these results, we choose to model the liquid base flow with simple parabolic velocity profiles for $U$ and $W$ in the inviscid stability analysis. In order to study a possible influence of the interface velocity (which is expected to increase when the profile develops downstream of the injection), the interface velocities can be set to arbitrary $U_{i}$ and $W_{i}$ by stretching the parabola.

\section{B. Results}

We first consider the case $\alpha=65.7^{\circ}, H_{l}=0.7 \mathrm{~mm}, U_{l}=1 \mathrm{~m} / \mathrm{s}$ (note that $U_{l}$ denotes the mean liquid velocity, and not the maximum velocity which is higher) and $U_{g}=80 \mathrm{~m} / \mathrm{s}$. We assume $W_{l} / U_{l}=3.5$, and parabolic velocity profiles in the liquid phase. Figure 17 left shows the results obtained for axisymmetric mode $n=0$ (solid line) and helical mode $n=1$ (dotted line), when the interface velocities $U_{i}$ and $W_{i}$ are taken equal to zero. The initial zero velocity at the interface models the velocity defect induced by the splitter plate. The growth rate is significantly larger for the axisymmetric mode. The most unstable wavenumber corresponds to $k \approx 4800 \mathrm{~m}^{-1}$, i.e., $\lambda \approx$ $1.3 \mathrm{~mm}$ : though we have not carried out measurements of wavelength, this order of magnitude is consistent with the visualizations of Figure 5, where the whole length corresponds to $l_{R}=11.4 \mathrm{~mm}$, 

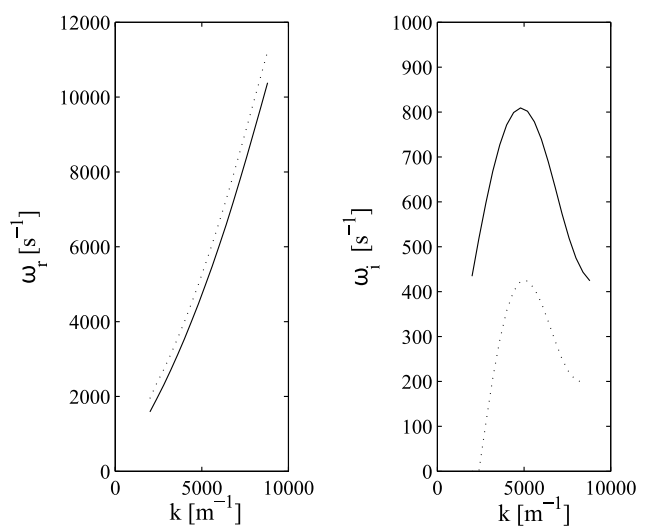

(a)
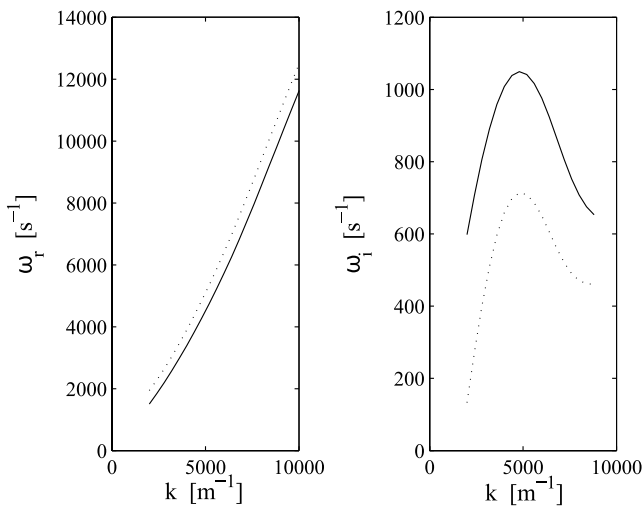

(b)

FIG. 17. Frequency and temporal growth rate predicted by inviscid analysis: $U_{g}=80 \mathrm{~m} / \mathrm{s}, U_{l}=1 \mathrm{~m} / \mathrm{s}, W_{l}=3.5 \mathrm{~m} / \mathrm{s}$. Solid line corresponds to $n=0$, and dotted line to $n=1$. (a) $U_{i}=0$ and $W_{i}=0$. (b) $U_{i}=U_{l} / 2$ and $W_{i}=W_{l} / 2$.

and wavelengths can be seen to be of the order of $l_{R} / 10$. Most unstable frequency corresponds to $f$ $\approx 711 \mathrm{~Hz}$, slightly larger but close to the frequency for $U_{g}=80 \mathrm{~m} / \mathrm{s}$ on Figure 13 . If the interface velocity is increased to $U_{i}=U_{l} / 2$ and $W_{i}=W_{l} / 2$, most unstable wavelength is unchanged, but frequency is slightly decreased to $f \approx 681 \mathrm{~Hz}$, and temporal growth rate is slightly increased by about $20 \%$ (Figure 17 right).

We then look at the influence of the swirl number on the wavelength and frequency of this most unstable mode. Gas and liquid velocities are kept fixed at, respectively, $U_{g}=80 \mathrm{~m} / \mathrm{s}$ and $U_{l}=1 \mathrm{~m} / \mathrm{s}$, and swirl is decreased from $W_{l} / U_{l}=3.5$ down to $W_{l} / U_{l}=2.5$. Figure 18 shows that when swirl is decreased the temporal growth rate rapidly decreases, and reaches zero for $S_{w} \approx 2.4$. Frequency decreases also significantly when swirl is reduced. This is not consistent with experiments, where the unstable mode is observed down to $S_{w}=0\left(\alpha=0^{\circ}\right)$, and where frequency does not significantly depend on the injection angle.

We then compute the wavelength and frequency of the most unstable mode as a function of gas velocity, for fixed interface velocity $U_{i}=U_{l} / 2$ and for a fixed swirl number $S_{w}=2.5$ (expected to approximately match the conditions of Figure 12(c)). Figure 19 shows that frequency and temporal growth rate are unaffected by gas velocity: this is contrary to the trend observed in experiments,
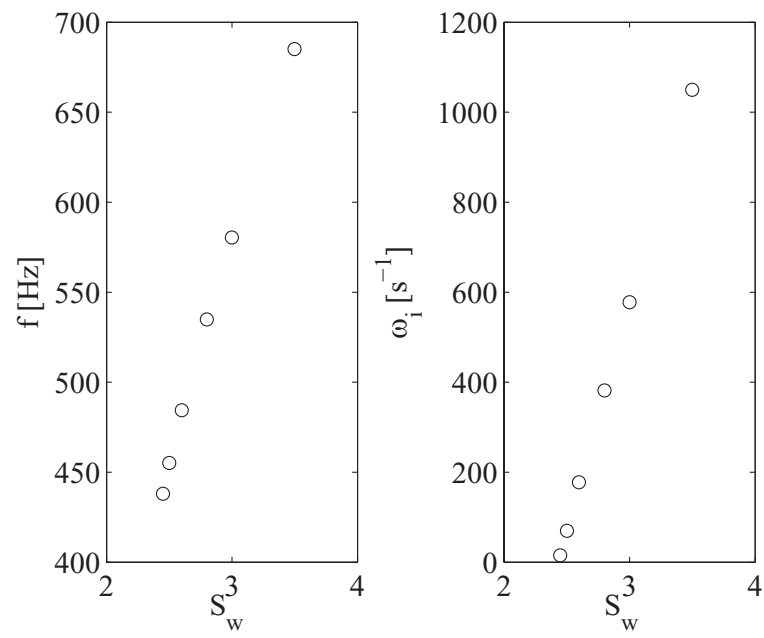

FIG. 18. Frequency (left) and temporal growth rate (right) as a function of swirl number, for fixed $U_{g}=80 \mathrm{~m} / \mathrm{s}, U_{l}=1 \mathrm{~m} / \mathrm{s}$, and $n=0$ (axisymmetric mode), inviscid analysis. 

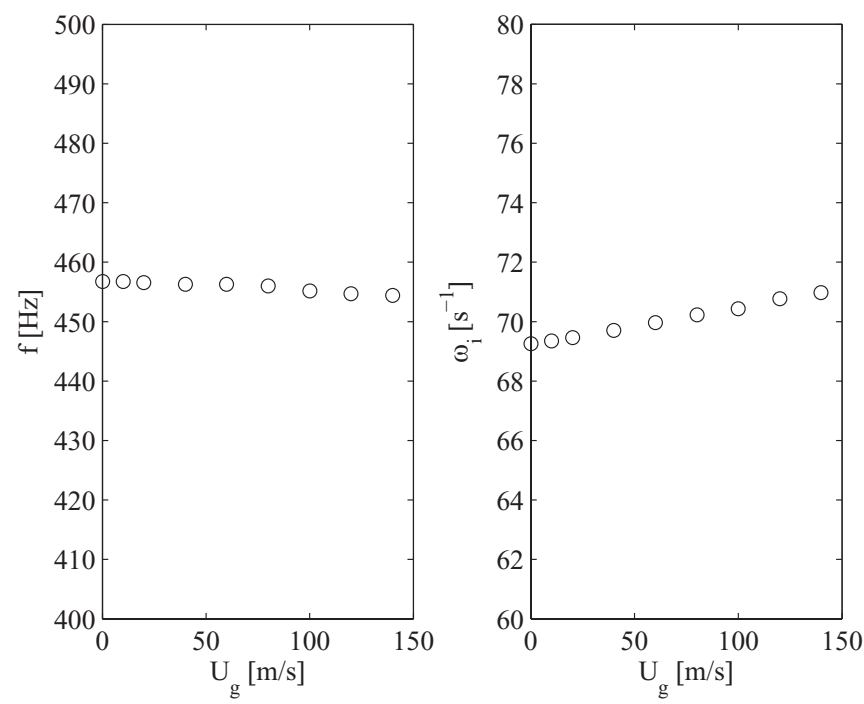

FIG. 19. Frequency (left) and temporal growth rate (right) as a function of gas velocity, for fixed $S_{w}=2.5, U_{l}=1 \mathrm{~m} / \mathrm{s}$, and $n=0$ (axisymmetric mode), inviscid analysis.

where frequency consistently increases with gas velocity. This suggests that the instability captured by the present inviscid stability analysis is not a shear instability. Also note that setting surface tension to zero has almost no effect on the graphs of Figure 17, except a slight reduction in temporal growth rate.

In order to help us determine the nature of the instability, we compute the rate of energy transfer via Reynolds stresses $u v \frac{d U}{d r}$ within the liquid stream: this term is positive when energy is taken from the base flow to feed the kinetic energy of the perturbation. Figure 20 shows that this contribution is positive next to the solid wall (its contribution next to the interface is negligible). This leads us to believe that the inviscid mode captured here is a Taylor-Couette mode arising from the boundary layer for the azimuthal velocity $W$ : this would be consistent with the fact that this mode disappears when swirl is decreased, and that it is roughly insensitive to the axial gas velocity. In addition, axisymmetric modes are known to be more unstable than helical ones for Taylor-Couette instability, as was observed on Figure 17.

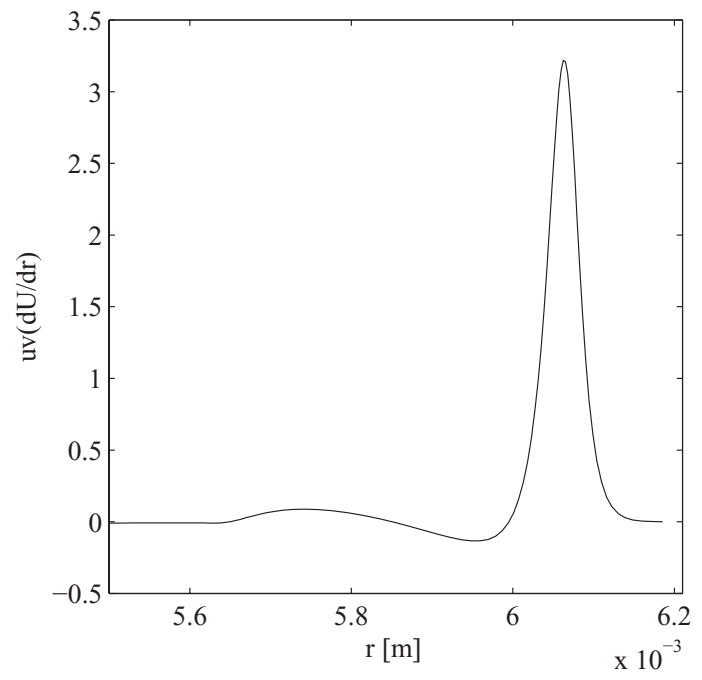

FIG. 20. Local energy transfer via Reynolds stresses in the liquid film: destabilization occurs next to the solid wall (located at $r=6.2 \mathrm{~mm}$ ). Computation for $U_{g}=80 \mathrm{~m} / \mathrm{s}, U_{l}=1 \mathrm{~m} / \mathrm{s}, S_{w}=3.5, n=0$, and $H_{l}=0.7 \mathrm{~mm}$. 


\section{VISCOUS LINEAR STABILITY ANALYSIS}

\section{A. Equations}

We now include viscosity in the stability analysis. In order to facilitate the resolution of the resulting equations, we will consider a zero swirl velocity for the base flow in this section, $W_{l}=0$, a hypothesis strictly valid when $\alpha=0^{\circ}$ : this approach is motivated by the fact that experiments show that swirl angle does not impact the frequency of the instability. We only look for axisymmetric modes $n=0$. The resulting system of equations is then

$$
\begin{gathered}
i \rho(-\omega+k U) \tilde{u}+\rho \tilde{v} \frac{d U}{d r}=-i k \tilde{p}+v\left[\frac{d \tilde{u}^{2}}{d r^{2}}-\tilde{u} k^{2}+\frac{1}{r} \frac{d \tilde{u}}{d r}\right], \\
i \rho(-\omega+k U) \tilde{v}=-\frac{d \tilde{p}}{d r}+v\left[\frac{d \tilde{v}}{d r^{2}}-\tilde{v}\left(\frac{1}{r^{2}}\right)+\frac{1}{r} \frac{d \tilde{v}}{d r}\right]+\sigma \frac{\tilde{\eta}}{R^{2}}\left(1-n^{2}-k^{2} R^{2}\right) \delta(r), \\
i \rho(-\omega+k U) \tilde{w}=v\left[\frac{d \tilde{w}}{d r^{2}}-\tilde{w}\left(\frac{1}{r^{2}}+k^{2}\right)+\frac{1}{r} \frac{d \tilde{w}}{d r}\right], \\
i \rho(-\omega+k U) \tilde{\eta}=\tilde{v}, \\
i k \tilde{u}+\frac{\tilde{v}}{r}+\frac{d \tilde{v}}{d r}=0,
\end{gathered}
$$

where $U, v$, and $\rho$ are to be set to $U_{g}, v_{g}$, and $\rho_{g}$ in the gas phase, and to $U_{l}, v_{l}$, and $\rho_{l}$ in the liquid phase. Following Heaton, ${ }^{16}$ we introduce the stream function $\phi$, from which axial and radial velocities can be recovered by

$$
\tilde{u}=\frac{1}{r} \frac{d \phi}{d r}, \quad \tilde{v}=-\frac{i k}{r} \phi,
$$

and the governing equation for $\phi$ is then analogous to the Orr-Sommerfeld equation

$$
\begin{aligned}
(U k-\omega) & \left(\phi^{\prime \prime}-\frac{\phi^{\prime}}{r}-k^{2} \phi\right)+\phi k\left(\frac{U^{\prime}}{r}-U^{\prime \prime}\right) \\
= & \frac{-i}{R e}\left[\phi^{\prime \prime \prime \prime}-\frac{2}{r} \phi^{\prime \prime \prime}+\frac{3}{r^{2}} \phi^{\prime \prime}-\frac{3}{r^{3}} \phi^{\prime}-2 k^{2}\left\{\phi^{\prime \prime}-\frac{\phi^{\prime}}{r}\right\}+k^{4} \phi\right],
\end{aligned}
$$

where a prime denotes differentiation to $r$. Boundary conditions are enforced at the outer wall: $\phi(R)$ $=0$ and $\phi^{\prime}(R)=0$, as well as on the axis where radial velocity $v$ has to vanish and axial velocity $u$ has to remain finite leading to $\phi(0)=0$ and $\phi^{\prime}(0)=0$. Two solutions $\phi_{1 l}$ and $\phi_{2 l}$ are integrated in the liquid phase from the outer wall to the interface, and two solutions $\phi_{1 g}$ and $\phi_{2 g}$ are integrated in the gas phase from the centerline to the interface. These two couples of solutions are connected at the interface $r=R-H_{l}$ via continuity of normal and tangential velocity, and continuity of normal and tangential stresses

$$
\begin{array}{r}
\phi_{l}=\phi_{g}, \\
\phi_{g}^{\prime}-\phi_{l}^{\prime}=\frac{k \phi_{g}}{k U_{i}-\omega}\left(U_{g}^{\prime}-U_{l}^{\prime}\right), \\
\mu_{g}\left(\phi_{g}^{\prime \prime \prime}-\frac{\phi_{g}^{\prime \prime}}{r_{i}}\right)-\phi_{g}^{\prime}\left[i \rho_{g}\left(k U_{i}-\omega\right)-\frac{\mu_{g}}{r_{i}^{2}}+3 \mu_{g} k^{2}\right] \\
+\phi_{g}\left(i k \rho_{g} U_{g}^{\prime}+2 \mu_{g} \frac{k^{2}}{r_{i}}\right)+i \sigma \frac{k^{2}}{r_{i}^{2}} \frac{1}{k U_{i}-\omega}\left(1-k^{2} r_{i}^{2}\right)= \\
\mu_{l}\left(\phi_{l}^{\prime \prime \prime}-\frac{\phi_{l}^{\prime \prime}}{r_{i}}\right)-\phi_{l}^{\prime}\left[i \rho_{l}\left(k U_{i}-\omega\right)-\frac{\mu_{l}}{r_{i}^{2}}+3 \mu_{l} k^{2}\right]+\phi_{l}\left(i \rho_{l} k U_{l}^{\prime}+2 \mu_{l} \frac{k^{2}}{r_{i}}\right),
\end{array}
$$




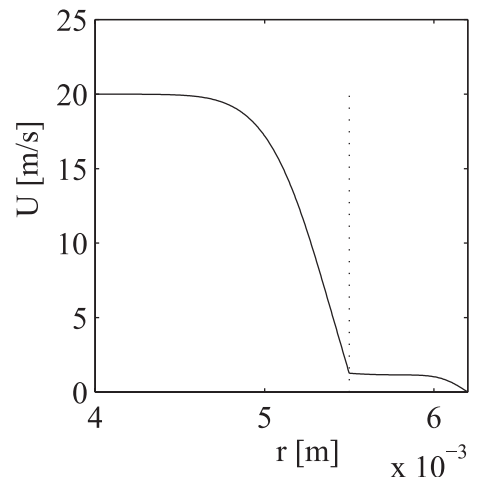

(a)

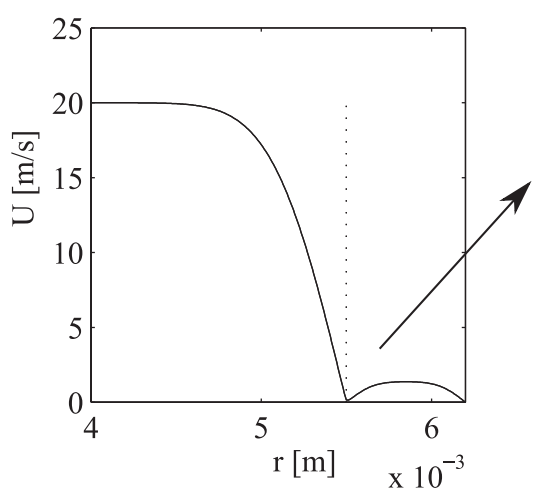

(b)

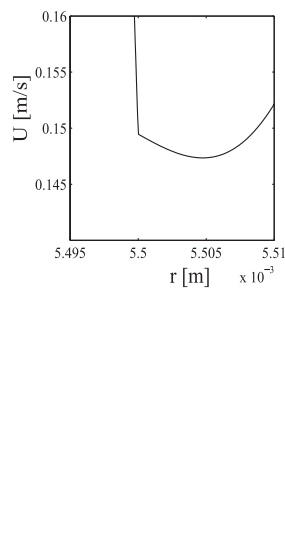

FIG. 21. Velocity profile used in the viscous linear stability analysis, for $U_{g}=20 \mathrm{~m} / \mathrm{s}, U_{l}=1 \mathrm{~m} / \mathrm{s}$, and $H_{l}=0.7 \mathrm{~mm}$. The liquid/gas interface is indicated by the vertical dotted line: (a) $\delta_{d}=\delta_{l}$; (b) $\delta_{d}=0.1 \delta_{l}$.

$$
\mu_{g}\left[k^{2} \phi_{g}+\frac{k U_{i}^{\prime \prime}}{\omega-k U} \phi_{g}+\phi_{g}^{\prime \prime}-\frac{\phi_{g}^{\prime}}{r_{i}}\right]=\mu_{l}\left[k^{2} \phi_{l}+\frac{k U_{i}^{\prime \prime}}{\omega-k U} \phi_{l}+\phi_{l}^{\prime \prime}-\frac{\phi_{l}^{\prime}}{r_{i}}\right],
$$

where $\mu_{g}$ and $\mu_{l}$ are gas and liquid dynamic viscosities, and $r_{i}=R-H_{l}$ stands for the interface location. Functions $\phi_{g}$ and $\phi_{l}$ denote the respective total stream functions in the gas and liquid phase. Stream functions and their derivatives are all evaluated for $r=r_{i}$. Similarly, $U_{g}^{\prime}, U_{l}^{\prime}, U_{g}^{\prime \prime}$, and $U_{l}^{\prime \prime}$ represent the first and second derivatives of the base flow respective gas and liquid velocities evaluated at the interface.

The base flow has to satisfy a similar set of conditions: continuity of normal and tangential velocities, and continuity of normal and tangential stresses. We chose the velocity profile suggested by Otto et al. ${ }^{17}$ constructed with a sum of error functions

$$
\left\{\begin{array}{l}
U_{g}(r)=U_{g} \operatorname{erf}\left(\frac{r_{i}-r}{\delta_{g}}\right)+U_{i}\left[1-\operatorname{erf}\left(\frac{r_{i}-r}{\delta_{d}}\right)\right], \\
U_{l}(r)=\left[U_{l m a x} \operatorname{erf}\left(\frac{r-r_{i}}{\delta_{l}}\right)+U_{i}\left[1-\operatorname{erf}\left(\frac{r-r_{i}}{\delta_{d}}\right)\right]\right] \operatorname{erf}\left(\frac{R-r}{\delta_{l}}\right),
\end{array}\right.
$$

where term $\operatorname{erf}\left[(R-r) / \delta_{l}\right]$ has been added to account for the boundary layer against the outer wall. Due to this boundary layer, $U_{\operatorname{lmax}}$ has to be slightly larger than $U_{l}$ to ensure that the mean liquid velocity remains equal to the desired value $U_{l}$. The magnitude of the velocity defect close to the interface is directly controlled by coefficient $\delta_{d}$ : for $\delta_{d}=\delta_{l}$ there is no velocity defect and the velocity profile is monotonous, while for $\delta_{d}<\delta_{l}$ a minimum velocity is reached close to the interface on the liquid phase. ${ }^{17}$ This velocity profile ensures continuity of shear stress provided that ${ }^{17}$

$$
U_{i}=\frac{\frac{\mu_{g} U_{g}}{\delta_{g}}+\frac{\mu_{l} U_{l \max }}{\delta_{l}}}{\mu_{g}+\mu_{l}} \delta_{d} .
$$

This profile is plotted on Figure 21 for two cases, $\delta_{d}=\delta_{l}$ (no velocity defect) and $\delta_{d}=0.1 \delta_{l}$ (strong velocity defect). Numerical integration of stream functions is carried out in Fortran, with a classical Runge-Kutta order 4-5 algorithm. Each couple of stream functions is orthonormalized during integration to ensure they remain independent. ${ }^{15}$

We chose to look for temporal modes, i.e., to solve the dispersion relation obtained from system of Eqs. (10)-(13) for complex $\omega$ for a fixed real wavenumber $k$. Figure 22(a) shows the unstable mode observed for air and water at $U_{g}=80 \mathrm{~m} / \mathrm{s}$ and $U_{l}=1 \mathrm{~m} / \mathrm{s}$ (conditions of Figure 17). We have chosen $\delta_{l}=H_{l} / 4$. Solid line shows results for $\delta_{d}=0.1 \delta_{l}$ (velocity defect) and dotted line for $\delta_{d}=\delta_{l}$ (no velocity defect). Note that the mean velocity $U_{l}$ is kept fixed, meaning that maximum velocity is necessarily different for both cases $\left(U_{\operatorname{lmax}}=1.39\right.$ for $\delta_{d}=0.1 \delta_{l}$ and $U_{l \max }=1.1$ for $\left.\delta_{d}=\delta_{l}\right)$. The 

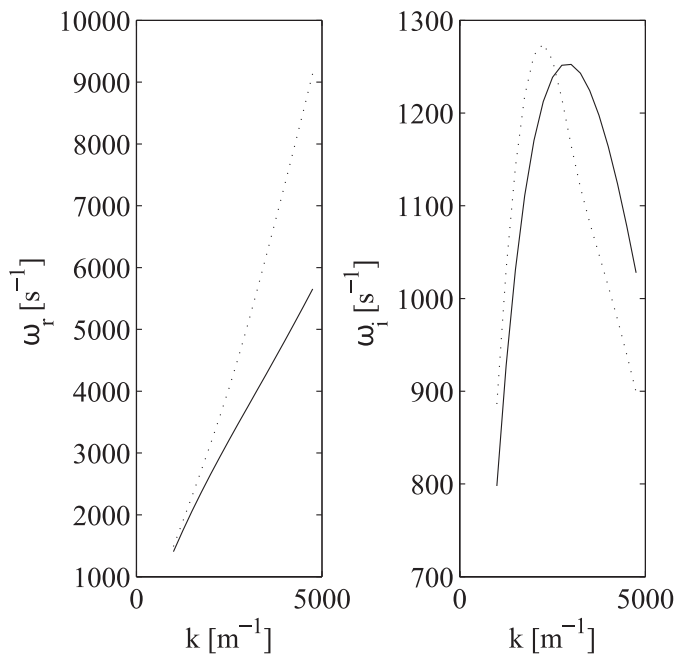

(a)

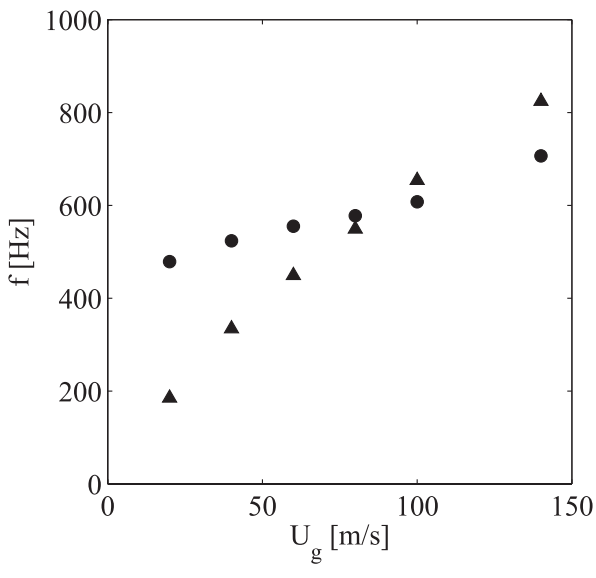

(b)

FIG. 22. (a) Unstable mode for $U_{g}=80 \mathrm{~m} / \mathrm{s}, H_{l}=0.7 \mathrm{~mm}$, and $U_{l}=1 \mathrm{~m} / \mathrm{s}$ : solid line $\delta_{d}=0.1 \delta_{l}$ and dotted line $\delta_{d}=\delta_{l}$; (b) frequency of the most unstable mode for varying gas velocities and fixed $U_{l}=1 \mathrm{~m} / \mathrm{s}$. $\bullet: \delta_{d}=0.1 \delta_{l} ; \boldsymbol{\Delta}: \delta_{d}=\delta_{l}$. Viscous analysis, $S_{w}=0$.

inclusion of a velocity defect increases slightly the wavenumber, but has no effect on the frequency since the increase in $k$ is compensated almost exactly by the decrease in velocity (decrease of slope on Figure 22(a) left). When gas velocity is increased we observe that the frequency of the mode strongly increases. Figure 22(b) shows frequency results as a function of $U_{g}$ : without a velocity deficit (symbol $\boldsymbol{\Delta}$ ) frequency is roughly proportional to gas velocity. When a velocity defect is included $\left(\delta_{d}=0.1 \delta_{l}\right.$, symbol $\bullet$ ) this trend is modified: frequency for zero gas velocity converges towards a finite frequency of around $400 \mathrm{~Hz}$. We next compare these results with experimental results: Figure 23 shows the superposition of experimental data from Figure 13 to predictions of Figure 22(b). Frequency is correctly predicted under the assumption $\delta_{d}=\delta_{l}$ corresponding to black triangles (no velocity defect).

Figure 24 shows the predicted wavelength and temporal growth rate of the instability as a function of gas velocity. Gas boundary layer thickness $\delta_{g}$ is set to the experimental value, which

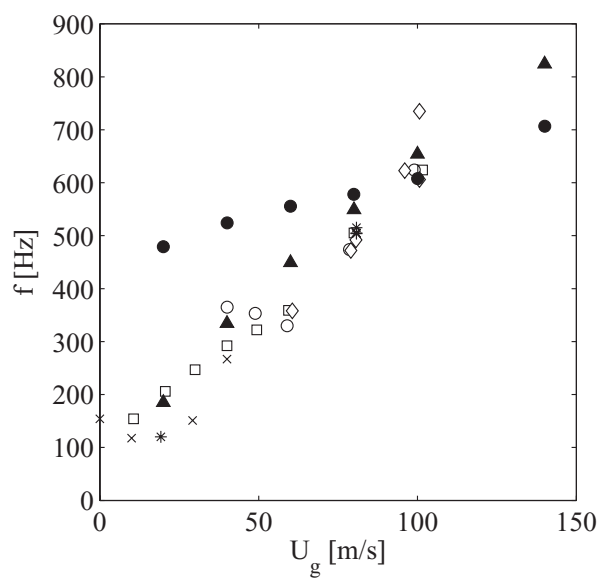

FIG. 23. Comparison of predictions of Figure 22 to experimental data of Figure 13(b), all for $H_{l}=0.7 \mathrm{~mm}$ and $U_{l}=1 \mathrm{~m} / \mathrm{s}$. Symbols are the same as in Figures 13 and 22, with filled symbols corresponding to viscous prediction. Frequency is correctly predicted under the assumption $\delta_{d}=\delta_{l}$ corresponding to black triangles (no velocity defect). 

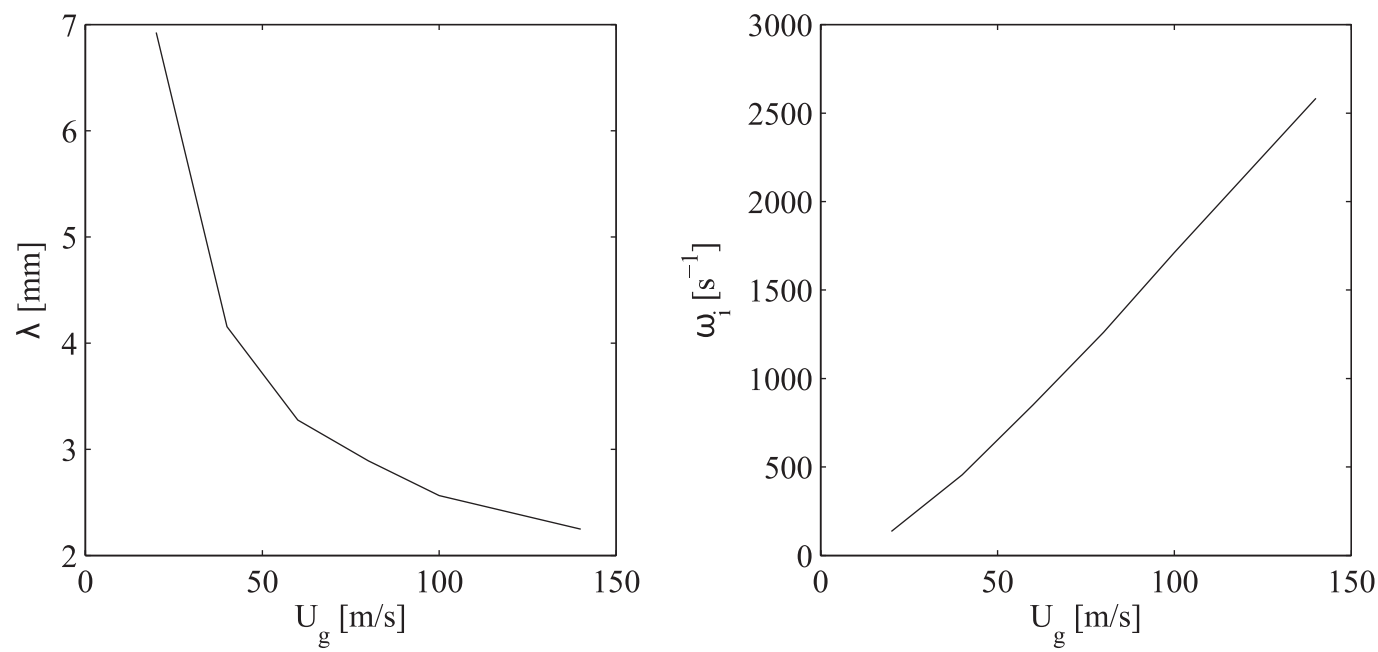

FIG. 24. Left: Predicted wavelength of the instability for varying gas velocity. Right: Predicted temporal growth rate as a function of gas velocity. Fixed liquid velocity $U_{l}=1 \mathrm{~m} / \mathrm{s}, H_{l}=0.7 \mathrm{~mm}$, no velocity deficit $\left(\delta_{d}=\delta_{l}\right)$.

varies between 479 and $367 \mu \mathrm{m}$ when $U_{g}$ increases from 20 to $140 \mathrm{~m} / \mathrm{s}$. Though wavelength could not be measured experimentally, we observe that the order of magnitude of the predicted wavelength is consistent with visualizations: $\lambda$ is typically of the order of a few millimeters. We also note that gas velocity has a strong impact on the temporal growth rate, which increases almost linearly with $U_{g}$.

In order to compare amplitude measurements of Figure 14 to predictions, we can solve the dispersion relation for a spatial solution: this yields directly a spatial growth rate $k_{i}$. Table II shows the results of both spatial and temporal analyses for the set of gas velocities corresponding to Figure 14 and a fixed liquid velocity $U_{l}=1 \mathrm{~m} / \mathrm{s}$. We can see that Gaster's relation ${ }^{18}$ gives a good estimate of the spatial growth rate for low gas velocities, but underestimates it for $U_{g}=80 \mathrm{~m} / \mathrm{s}$. Figure 25 shows a comparison of these predictions with experimental data for $\alpha=0^{\circ}$ and three gas velocities. The agreement is not good for the lowest gas velocity $\left(U_{g}=20 \mathrm{~m} / \mathrm{s}\right)$ : predicted growth rate is much smaller than observed. Note also that for $U_{g}=0 \mathrm{~m} / \mathrm{s}$ stability analysis predicts that the spatial growth rate tends to zero, whereas in experiments growth for $U_{g}=0 \mathrm{~m} / \mathrm{s}$ and $U_{g}=$ $20 \mathrm{~m} / \mathrm{s}$ is roughly similar, and seems to reach a plateau (Figure 14). Nonetheless, slopes for $U_{g}=$ $40 \mathrm{~m} / \mathrm{s}$ (dashed line) and $U_{g}=80 \mathrm{~m} / \mathrm{s}$ (dashed-dotted line) are in relatively good agreement with experimental slopes close to injection, i.e., for $z<2 \mathrm{~mm}$ for $U_{g}=40 \mathrm{~m} / \mathrm{s}$ and $z<1 \mathrm{~mm}$ for $U_{g}=$ $80 \mathrm{~m} / \mathrm{s}$.

We now turn to the question of the nature of this instability: the fact that frequency and growth rate are strongly impacted by gas velocity suggests that it is a shear instability. Figure 26 shows the variation of the norm of the temporal eigenfunction $\phi$ in the case $\delta_{d}=\delta_{l}$ (no velocity defect)

TABLE II. Comparison of results from temporal and spatial viscous linear stability analyses $\left(U_{l}=1 \mathrm{~m} / \mathrm{s}, H_{l}=0.7 \mathrm{~mm}\right.$, air and water); $v_{g}$ stands for group velocity.

\begin{tabular}{lccccr}
\hline \hline & $U_{g}(\mathrm{~m} / \mathrm{s})$ & $k\left(\mathrm{~m}^{-1}\right)$ & $\omega_{r}\left(\mathrm{~s}^{-1}\right)$ & $\omega_{i}\left(\mathrm{~s}^{-1}\right)$ & $\omega_{i} / v_{g}$ \\
\hline Temporal & 20 & 907 & 1162 & 136 & 95 \\
& 40 & 1512 & 2101 & 456 & 328 \\
& 80 & 2174 & 3451 & 1261 & 680 \\
& $U_{g}$ & $k_{r}$ & $\omega$ & $k_{i}$ & \\
\hline Spatial & 20 & 1195 & 1500 & 355 & \\
& 40 & 2016 & 3000 & 922 & \\
& 80 & & &
\end{tabular}




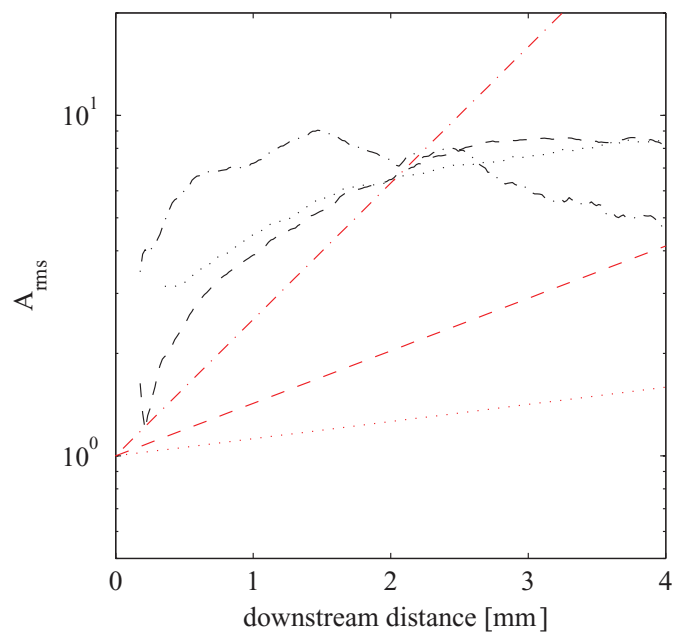

FIG. 25. Comparison of experimentally measured amplitude of the surface perturbation ( $r m s$ value of amplitude in pixels) and spatial growth rate predicted by spatial stability analysis. Fixed $U_{l}=1 \mathrm{~m} / \mathrm{s}, \alpha=0^{\circ}\left(S_{w}=0\right)$, and $H_{l}=0.7 \mathrm{~mm}$. Dotted line: $U_{g}=20 \mathrm{~m} / \mathrm{s}$; dashed line: $U_{g}=40 \mathrm{~m} / \mathrm{s}$; dashed-dotted line: $U_{g}=80 \mathrm{~m} / \mathrm{s}$.

and for two gas velocities: in both cases, the function reaches its maximum in the strong shear regions on both side of the interface, but appears unaffected by the outer wall boundary layer. As mentioned in Sec. III B, a simple scaling law based on an inviscid linear stability analysis has been suggested by Marmottant and Villermaux: ${ }^{12}$ it predicts $f \sim \frac{\rho_{g}}{\rho_{l}} \frac{U_{g}}{\delta_{g}}$, an estimate much lower than in our experiments as discussed in Sec. III B. In order to test the corresponding scaling law, and to discern better the physics involved here we have looked at the influence of several parameters on the most unstable mode, and show the results in Table III. Conditions were varied around a chosen set of data: $U_{g}=80 \mathrm{~m} / \mathrm{s}, U_{l}=1 \mathrm{~m} / \mathrm{s}, H_{l}=0.7 \mathrm{~mm}$ for air and water at room temperature. Not shown in this table are runs for varying gas kinematic viscosity: this parameter has no impact on the unstable mode. Similarly, changing the extent or even suppressing the liquid boundary layer against the outer solid wall has no effect on the mode considered here. All results of Table III are obtained for

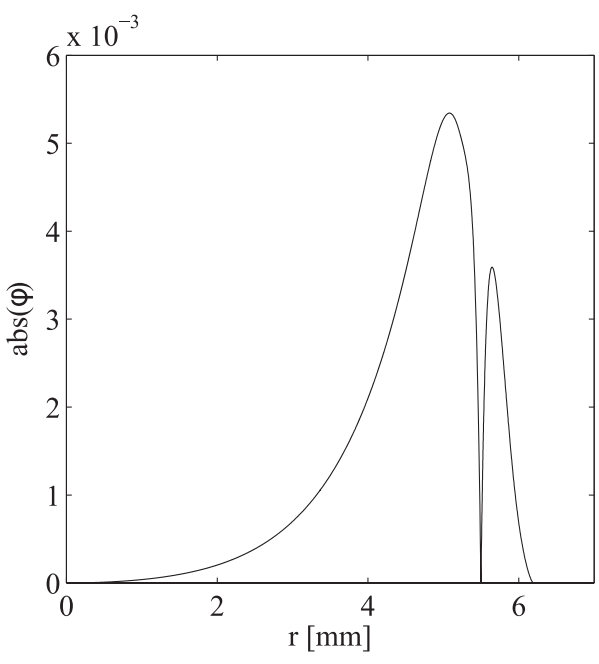

(a)

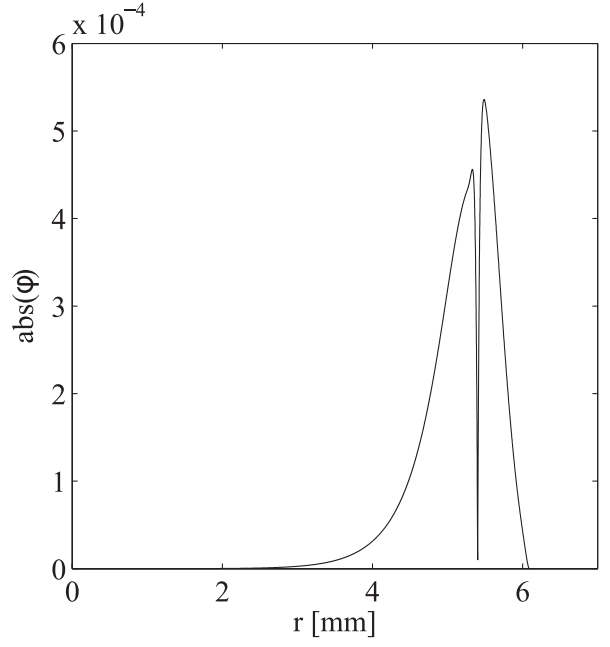

(b)

FIG. 26. Eigenfunction $\phi$ for $U_{l}=1 \mathrm{~m} / \mathrm{s}, H_{l}=0.7 \mathrm{~mm}$, and $\delta_{d}=\delta_{l}$ and for two gas velocities: (a) $U_{g}=20 \mathrm{~m} / \mathrm{s}$; (b) $U_{g}=$ $80 \mathrm{~m} / \mathrm{s}$. 
TABLE III. Results from temporal viscous linear stability analysis.

\begin{tabular}{|c|c|c|c|c|c|c|c|c|c|c|c|}
\hline $\begin{array}{l}U_{g} \\
(\mathrm{~m} / \mathrm{s})\end{array}$ & $\begin{array}{c}U_{l} \\
(\mathrm{~m} / \mathrm{s})\end{array}$ & $\begin{array}{c}\delta_{g} \\
(\mu \mathrm{m})\end{array}$ & $\begin{array}{c}\sigma \\
(\mathrm{N} / \mathrm{m})\end{array}$ & $\begin{array}{c}v_{l} \\
(\mathrm{cSt})\end{array}$ & $\begin{array}{c}\rho_{g} \\
\left(\mathrm{~kg} / \mathrm{m}^{3}\right)\end{array}$ & $\begin{array}{c}\rho_{l} \\
\left(\mathrm{~kg} / \mathrm{m}^{3}\right)\end{array}$ & $\begin{array}{c}H_{l} \\
(\mathrm{~mm})\end{array}$ & $\begin{array}{c}U_{i} \\
(\mathrm{~m} / \mathrm{s})\end{array}$ & $\begin{array}{c}k \\
\left(\mathrm{~m}^{-1}\right)\end{array}$ & $\begin{array}{c}\omega_{r} \\
\left(\mathrm{~s}^{-1}\right)\end{array}$ & $\begin{array}{c}\omega_{i} \\
\left(\mathrm{~s}^{-1}\right)\end{array}$ \\
\hline 40 & 1 & 393 & 0.072 & 1 & 1.2 & 1000 & 0.7 & 1.41 & 1590 & 2218 & 514 \\
\hline 80 & 1 & 393 & 0.072 & 1 & 1.2 & 1000 & 0.7 & 1.68 & 2220 & 3515 & 1275 \\
\hline 160 & 1 & 393 & 0.072 & 1 & 1.2 & 1000 & 0.7 & 2.22 & 2839 & 5351 & 2741 \\
\hline 80 & 0.5 & 393 & 0.072 & 1 & 1.2 & 1000 & 0.7 & 1.11 & 2220 & 2198 & 1330 \\
\hline 80 & 1 & 393 & 0.072 & 1 & 1.2 & 1000 & 0.7 & 1.68 & 2220 & 3515 & 1275 \\
\hline 80 & 2 & 393 & 0.072 & 1 & 1.2 & 1000 & 0.7 & 2.83 & 2235 & 6069 & 1189 \\
\hline 80 & 1 & 98 & 0.072 & 1 & 1.2 & 1000 & 0.7 & 3.34 & 3798 & 8149 & 4544 \\
\hline 80 & 1 & 196 & 0.072 & 1 & 1.2 & 1000 & 0.7 & 2.22 & 2835 & 5096 & 2542 \\
\hline 80 & 1 & 393 & 0.072 & 1 & 1.2 & 1000 & 0.7 & 1.71 & 2220 & 3515 & 1275 \\
\hline 80 & 1 & 786 & 0.072 & 1 & 1.2 & 1000 & 0.7 & 1.42 & 1596 & 2269 & 559 \\
\hline 80 & 1 & 393 & 0.018 & 1 & 1.2 & 1000 & 0.7 & 1.68 & 3330 & 5263 & 1587 \\
\hline 80 & 1 & 393 & 0.036 & 1 & 1.2 & 1000 & 0.7 & 1.68 & 2731 & 4286 & 1443 \\
\hline 80 & 1 & 393 & 0.072 & 1 & 1.2 & 1000 & 0.7 & 1.68 & 2220 & 3515 & 1275 \\
\hline 80 & 1 & 393 & 0.142 & 1 & 1.2 & 1000 & 0.7 & 1.68 & 1749 & 2730 & 1087 \\
\hline 80 & 1 & 393 & 0.072 & 0.6 & 1.2 & 1000 & 0.7 & 2 & 2046 & 2988 & 1090 \\
\hline 80 & 1 & 393 & 0.072 & 1 & 1.2 & 1000 & 0.7 & 1.68 & 2221 & 3515 & 1275 \\
\hline 80 & 1 & 393 & 0.072 & 2 & 1.2 & 1000 & 0.7 & 1.43 & 2490 & 3802 & 1429 \\
\hline 80 & 1 & 393 & 0.072 & 1 & 0.6 & 1000 & 0.7 & 1.42 & 1931 & 2771 & 823 \\
\hline 80 & 1 & 393 & 0.072 & 1 & 1.2 & 1000 & 0.7 & 1.68 & 2221 & 3515 & 1275 \\
\hline 80 & 1 & 393 & 0.072 & 1 & 2.4 & 1000 & 0.7 & 2.19 & 2268 & 3888 & 1790 \\
\hline 80 & 1 & 393 & 0.072 & 1 & 1.2 & 500 & 0.7 & 2.19 & 1916 & 3258 & 1609 \\
\hline 80 & 1 & 393 & 0.072 & 1 & 1.2 & 1000 & 0.7 & 1.68 & 2221 & 3515 & 1275 \\
\hline 80 & 1 & 393 & 0.072 & 1 & 1.2 & 2000 & 0.7 & 1.43 & 2460 & 3521 & 969 \\
\hline 80 & 1 & 393 & 0.072 & 1 & 1.2 & 1000 & 0.49 & 1.68 & 4824 & 2704 & 4429 \\
\hline 80 & 1 & 393 & 0.072 & 1 & 1.2 & 1000 & 0.56 & 1.68 & 3020 & 4145 & 1828 \\
\hline 80 & 1 & 393 & 0.072 & 1 & 1.2 & 1000 & 0.7 & 1.68 & 2221 & 3459 & 1277 \\
\hline 80 & 1 & 393 & 0.072 & 1 & 1.2 & 1000 & 1.4 & 1.68 & 1975 & 3132 & 1454 \\
\hline
\end{tabular}

$\delta_{d}=\delta_{l}$, i.e., no velocity defect in the base flow at the interface (conditions for which the agreement of predictions with experimental data was best).

Results of Table III show that though reducing $\delta_{g}$ increases $k$, the influence of $\rho_{l}$ on $k$ is not compatible with the inviscid scaling law $k \sim \sqrt{\rho_{g} / \rho_{l}} / \delta_{g}$ : doubling $\rho_{l}$ increases $k$, instead of reducing it. Next to $\delta_{g}$, the parameter having the most influence on $k$ appears to be $U_{g}$, whose effect on $k$ is exactly the opposite of $\delta_{g}$ : this suggests that both parameters probably influence $k$ via the gas shear rate $\gamma_{g}=U_{g} / \delta_{g}$. We also note a strong increase in $k$ when the liquid channel thickness $H_{l}$ is reduced (with $\delta_{l}$ kept constant), and when surface tension is decreased.

We believe this mode is akin to the family of shear modes discussed in Charru and Hinch, ${ }^{19}$ and initially evidenced by Yih, ${ }^{20}$ Hooper and Boyd, ${ }^{21}$ and Hooper and Boyd. ${ }^{22}$ These modes, whose mechanism depends on the jump in viscosity across the interface (see $\mathrm{Hinch}^{23}$ for a detailed discussion of its mechanism in the limit of short wavelengths), have recently been discussed in the context of liquid atomization by Boeck and Zaleski ${ }^{24}$ and Otto et al. ${ }^{17} \mathrm{We}$ think in the present situation the mode is strongly impacted by surface tension (see Table III) and channel thickness $H_{l}$ (of the order of $\lambda / 4$ ). Note also that previous analyses for this family of modes are for Couette flow: the wavelength found here is much larger than $\delta_{g}$, and this parameter may also affect the instability other than via the shear rate only.

Results of Table III show that though $U_{l}$ has no effect on the wavelength, doubling $U_{l}$ nearly doubles the frequency of the instability: close examination of the data for these conditions show that phase velocity of the most unstable mode is directly proportional to interface velocity $U_{i}$ (itself fixed by Eq. (14)). This holds for most conditions of Table III, except for the smaller $\delta_{g}$ investigated 


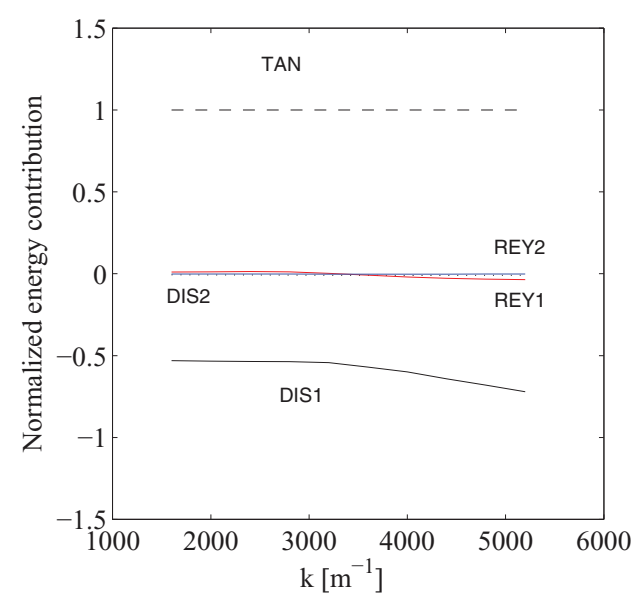

FIG. 27. Energy budget for $U_{g}=80 \mathrm{~m} / \mathrm{s}, U_{l}=1 \mathrm{~m} / \mathrm{s}, H_{l}=0.7 \mathrm{~mm}$, and $\delta_{g}=393 \mu \mathrm{m}$. The largest contribution to the instability is from term TAN, originating from the work of (viscous) tangential stresses.

(phase velocity smaller than $U_{i}$ ) and for the smaller $H_{l}$ investigated (phase velocity divided by three when $H_{l}$ is decreased by $30 \%$ ).

In order to support the claim that the unstable mode is a viscous mode we have carried out an energy budget following the method first suggested by Boomkamp and Miesen: ${ }^{25}$ we compute an energy budget for the temporal eigenmode corresponding to the reference conditions of Table III (second line), namely, air and water for $U_{g}=80 \mathrm{~m} / \mathrm{s}, U_{l}=1 \mathrm{~m} / \mathrm{s}, H_{l}=0.7 \mathrm{~mm}$, and $\delta_{g}=393 \mu \mathrm{m}$. The energy budget can be written as

$$
\frac{d E_{k i n}}{d t}=R E Y 1+R E Y 2+T A N+N O R+D I S 1+D I S 2,
$$

where $E_{k i n}$ is the total kinetic energy of the eigenmode (gas+liquid), $R E Y 1$ (resp. REY2) is the transfer of energy from the base flow to the perturbation via Reynolds stresses in the liquid (resp. gas) stream, TAN is the work of tangential stresses, NOR the contribution of normal stresses (surface tension in the present case), and DIS1 (resp. DIS2) the dissipation in the liquid phase (resp. gas phase). The expressions for each of these terms are given in Appendix B. We compute each term for a broad range of wavenumbers $k$, and normalize them by the sum of all positive terms (i.e., destabilizing terms). Figure 27 shows that the most destabilizing term is by far TAN, which corresponds to the work done by viscous stresses. Contribution $R E Y 1$ (red solid line), which corresponds to the contribution of Reynolds stresses in the liquid stream, is destabilizing for $k<3300$, but negligible compared to the viscous term. Contribution $R E Y 2$, blue curve, is negative (stabilizing) over the range of $k$ studied here. Most dissipation occurs in the liquid via DIS1. Normalized surface tension contribution NOR is not shown on Figure 27: it is negative, and smaller than $10^{-4}$ for the range of $k$ studied here. The most unstable $k$ for these conditions corresponds to $k=2220 \mathrm{~m}^{-1}$ (see Table III).

Results of Figures 3 and 12 also indicated the presence of a second lower frequency unstable mode for certain experimental conditions. This mode was not predicted by the present stability analysis. Our guess is that this may be an absolute instability: such a transition from a convective instability (i.e., noise amplification) to an absolute instability (akin to a resonance) has recently been evidenced on an air-water mixing layer, ${ }^{26}$ and the signature of this transition is precisely the apparition of sharp frequency peaks in spectra of the interface position. Absolute instability may be induced in the present configuration by the strong confinement in the recess zone. ${ }^{27}$

\section{CONCLUSION}

We have characterized the two-phase flow inside a gas-centered swirl coaxial injector by investigating the effect of swirl strength on the intact liquid length $L_{b}$. We have shown that the intact liquid length decreases as the swirl strength decreases and the dynamic pressure ratio increases. We 
have proposed a simple model to account for the influence of these parameters on $L_{b}$ : the predictions of this model are consistent with experiments, and suggest that the relevant parameter controlling $L_{b}$ is an effective dynamic pressure ratio $M /\left(1+S_{w}^{2} H_{l} / R\right)$. The relevance of this model supports the hypothesis that the decrease of $L_{b}$ when $M$ is increased is caused by increased acceleration of the liquid, and not by increased stripping of droplets from the liquid bulk as in classical mixing layer experiments. We think the present mechanism may also be relevant to applications involving atomization of $2 \mathrm{D}$ thin films.

A frequency instability is observed on the liquid issued from the injector. This frequency increases with gas velocity, and does not appear to depend on the initial swirl number. This frequency is much larger than the frequency observed in classical mixing layer experiments for similar air/water velocities. An inviscid stability analysis was not able to capture it. When viscosity is included in the analysis an instability is predicted with a frequency close to that of experiments. The order of magnitude of the predicted growth rate is in relative agreement with experiments, except at very low gas velocities. We believe this instability is related to the family of viscous shear instabilities described in Charru and Hinch, ${ }^{19}$ which occur when a jump in viscosity is present across the interface.

The fact that frequencies and wavenumbers are much larger here than on air/water mixing layer experiments carried out for similar gas and velocities may be related to the very small thickness $H_{l}$ of the liquid layer. Characterizing more precisely the influence of this parameter may be crucial to better predict the surface instability frequencies expected in applications related to drop formation.

\section{ACKNOWLEDGMENTS}

We thank Sylvain Marty, Antoine Delon, and Youngsun Yi for valuable help on the experimental setup and the image processing. We acknowledge financial support from the Egide/NRF PHC Star program. The LEGI laboratory is part of the LabEx Tec 21 (Investissements d'Avenir - Grant Agreement $n^{\circ}$ ANR-11-LABX-0030).

\section{APPENDIX A: IMAGE PROCESSING}

We detail here the image analysis method used for intact length measurement.

The 8-bit gray-scaled raw images captured by the digital camera were averaged and then converted into binary image files using a proper threshold value obtained via Otsu's method. This method calculates the threshold value which minimizes the interclass variance of the bi-modal histogram assumed from the gray level intensities $\left(\mathrm{Otsu}^{8}\right)$. Once the binary image has been computed, the slope of the intact liquid area is calculated by a simple linear regression with liquid interface points. The intact liquid length $L_{b}$ can then be derived from the cone angle $\beta$ (see Figure 28) by $L_{b}=H / \tan \beta$. Here, $H$ represents the height of liquid inlet flow at the liquid outlet. It can be seen from visualizations that this height is larger than the geometrical height $H_{l}$, but it is smaller than $H_{l}+0.3 \mathrm{~mm}\left(H_{l}+\right.$ lip thickness). The increase of the height is due to the low pressure in the gas flow recirculation behind the sheltering lip. Note however, that for the sake of simplification we still calculate the mean axial liquid velocity based on $H_{l}$. In order to improve the reliability of measurements, we have investigated the effects of the threshold level on the cone angle. Figure 29 shows a series of raw images, and the corresponding averaged image on the right. Randomly distributed white regions at the left of the liquid tongue on raw images are due to refraction effects caused by the corrugation of the liquid surface. Figure 30 then shows the binary images obtained from this averaged image for different threshold level varying from $40 \%$ to $100 \%$ of Otsu's value. At low threshold values, the binary images do not appropriately represent the intact liquid area because waves or fragmented liquid inclusions connected to the intact liquid area are retained, and impair slope calculation. On the other hand, for high threshold values, the intact liquid area quickly becomes partially perforated because of inhomogeneous light intensity inside the swirled liquid flow. In light of this, we decided to process our data with a threshold level of $80 \%$ of Otsu's value.

The length of the remaining liquid tongue after thresholding is $L_{0}$ : in order to prevent the liquid film present at the end of the intact liquid area from impairing linear regression, we chose to keep 

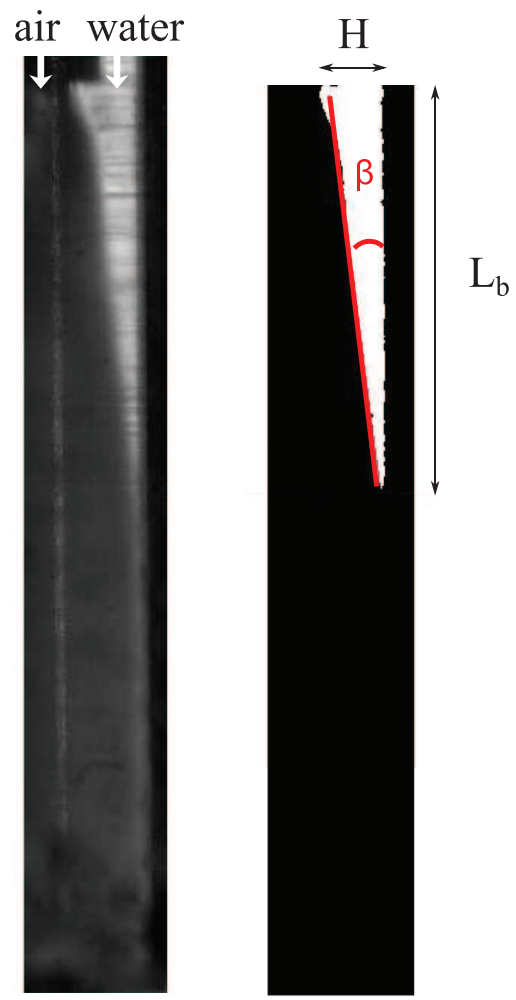

recess length

lR

FIG. 28. Illustration of image processing and definition of $\beta\left(H_{l}=0.5 \mathrm{~mm}, \alpha=0^{\circ}, M=2.07\right)$. Left: averaged raw image; right: thresholded image.

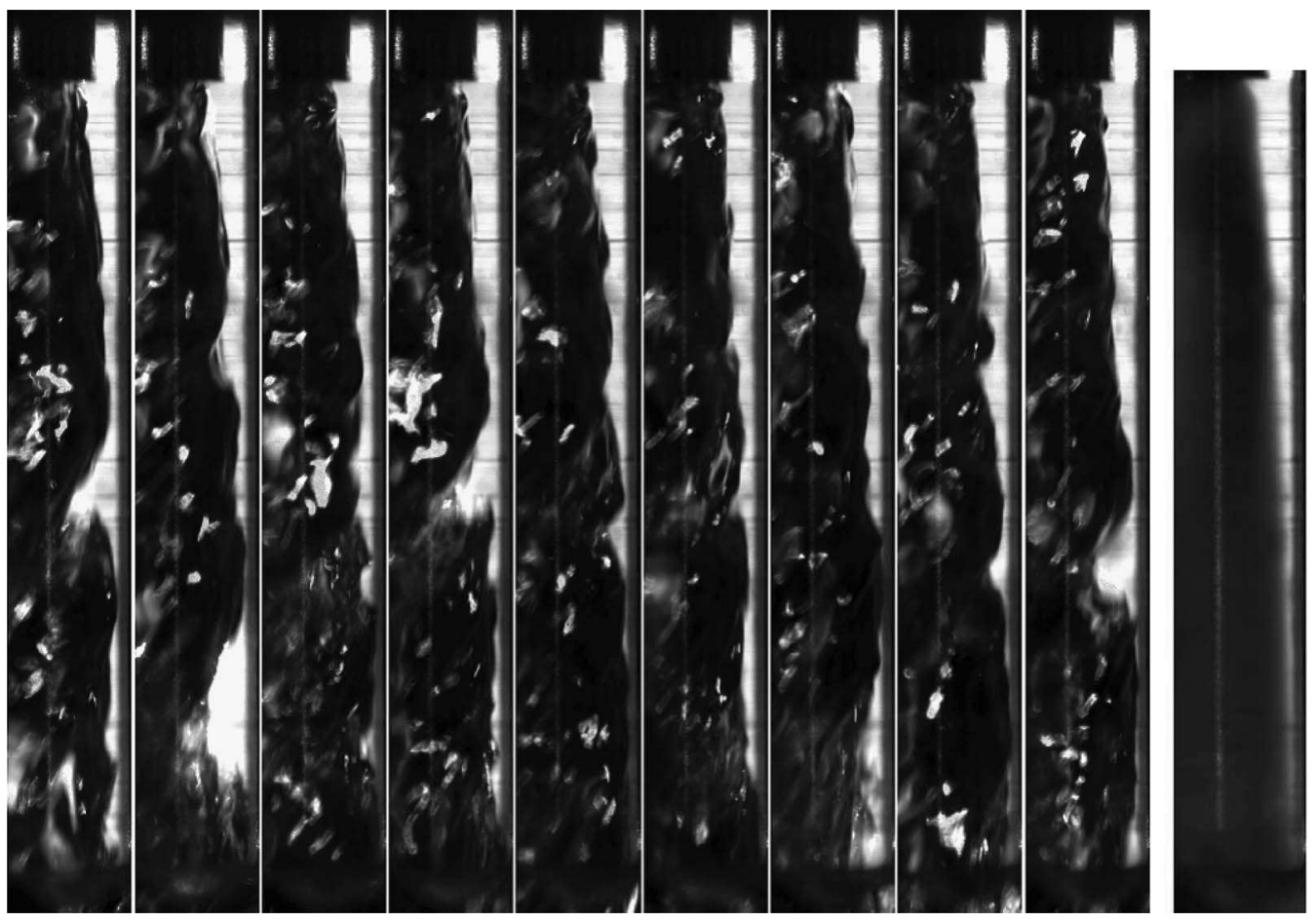

FIG. 29. Raw images, and resulting averaged image on the right $\left(H_{l}=0.5 \mathrm{~mm}, \alpha=65.7^{\circ}, U_{l}=1.4 \mathrm{~m} / \mathrm{s}, M=1.83\right)$. 


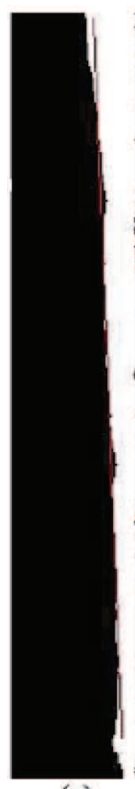

(a)

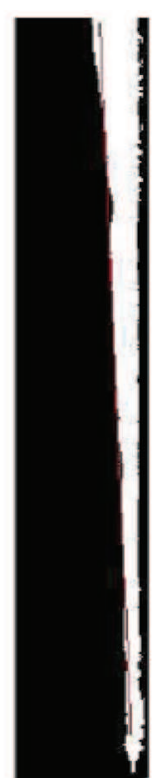

(b)

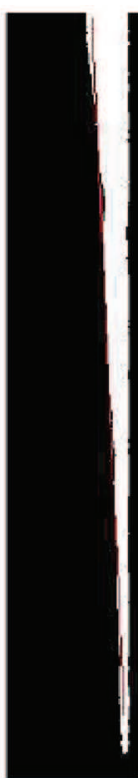

(c)

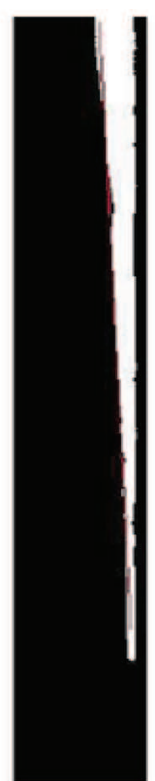

(d)

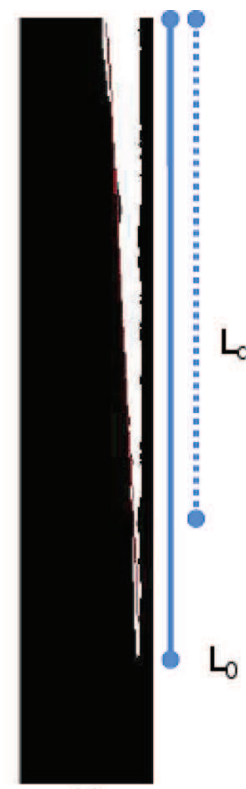

(e)

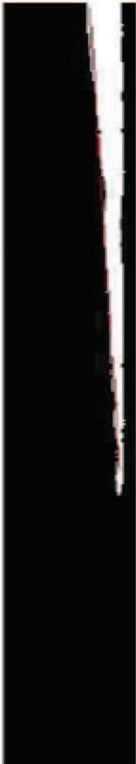

(f)

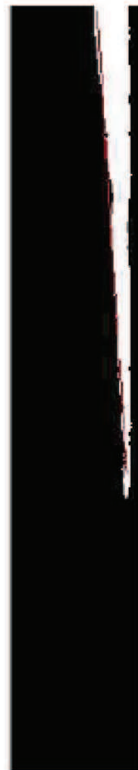

(g)

FIG. 30. Binary images as a function of the threshold level: $\%$ means the percentage of Otsu's value $\left(H_{l}=0.5 \mathrm{~mm}, \alpha=\right.$ $65.7^{\circ}, U_{l}=1.4 \mathrm{~m} / \mathrm{s}, M=1.83$ ): (a) $40 \%$, (b) $50 \%$, (c) $60 \%$, (d) $70 \%$, (e) $80 \%$, (f) $90 \%$, (g) $100 \%$.

only a fraction of $L_{0}$, corresponding to length $L_{c}$, for the interpolation of the liquid interface (see Figure 30). If $L_{c}$ is too close to $L_{0}$, the cone angle will be underestimated because of the liquid film present at the end of the intact liquid area. On the contrary, if $L_{c}$ is too short, the linear regression line may not capture correctly the slope of the liquid interface because the slope is calculated only over the forepart of the intact liquid area. We have chosen to carry out measurements with $L_{c}=$ $0.8 L_{0}$.

\section{APPENDIX B: ENERGY BUDGET}

We give here the expressions for each of the terms in Eq. (15). The expressions are valid for a temporal perturbation (real $k$ ), and correspond to an energy rate per unit axial length $z$.

$$
\begin{gathered}
\frac{d E_{k i n}}{d t}=\omega_{i}\left[\rho_{g} \int_{0}^{R-H_{l}}\left(\phi_{g}^{\prime 2}+k^{2} \phi_{g}^{2}\right) \frac{1}{r} d r+\rho_{l} \int_{R-H_{l}}^{R}\left(\phi_{l}^{\prime 2}+k^{2} \phi_{l}^{2}\right) \frac{1}{r} d r\right] \exp \left(2 \omega_{i}\right), \\
R E Y 1=\frac{i k \rho_{l}}{2}\left[\int_{R-H_{l}}^{R}\left(\phi_{l}^{\prime *} \phi_{l}-\phi_{l}^{*} \phi_{l}^{\prime}\right) \frac{1}{r} \frac{d U}{d r} d r\right] \exp \left(2 \omega_{i}\right), \\
R E Y 2=\frac{i k \rho_{g}}{2}\left[\int_{0}^{R-H_{l}}\left(\phi_{g}^{\prime *} \phi_{g}-\phi_{g}^{*} \phi_{g}^{\prime}\right) \frac{1}{r} \frac{d U}{d r} d r\right] \exp \left(2 \omega_{i}\right), \\
D I S 1=-\mu_{l}\left[\int _ { R - H _ { l } } ^ { R } \left(4 k^{2}\left|\phi_{l}^{\prime}\right|^{2}+\left|\phi_{l}^{\prime \prime}\right|^{2}+k^{2}\left(\phi_{l}^{\prime \prime *} \phi_{l}+\phi_{l}^{*} \phi_{l}^{\prime \prime}\right)+k^{4}\left|\phi_{l}\right|^{2}+4 k^{2} \frac{\left|\phi_{l}\right|^{2}}{r^{2}}\right.\right. \\
\left.\left.-3 \frac{k^{2}}{r}\left(\phi_{l}^{\prime *} \phi_{l}+\phi_{l}^{*} \phi_{l}^{\prime}\right)+\frac{\left|\phi_{l}^{\prime}\right|^{2}}{r^{2}}-\frac{1}{r}\left(\phi_{l}^{\prime \prime *} \phi_{l}^{\prime}+\phi_{l}^{\prime *} \phi_{l}^{\prime \prime}\right)\right) \frac{1}{r} d r\right] \exp \left(2 \omega_{i}\right), \\
{\left[\int _ { 0 } ^ { R - H _ { l } } \left(4 k^{2}\left|\phi_{g}^{\prime}\right|^{2}+\left|\phi_{g}^{\prime \prime}\right|^{2}+k^{2}\left(\phi_{g}^{\prime \prime *} \phi_{g}+\phi_{g}^{*} \phi_{g}^{\prime \prime}\right)+k^{4}\left|\phi_{g}\right|^{2}+4 k^{2} \frac{\left|\phi_{g}\right|^{2}}{r^{2}}\right.\right.} \\
\left.\left.-3 \frac{k^{2}}{r}\left(\phi_{g}^{\prime *} \phi_{g}+\phi_{g}^{*} \phi_{g}^{\prime}\right)+\frac{\left|\phi_{g}^{\prime}\right|^{2}}{r^{2}}-\frac{1}{r}\left(\phi_{g}^{\prime \prime *} \phi_{g}^{\prime}+\phi_{g}^{\prime *} \phi_{g}^{\prime \prime}\right)\right) \frac{1}{r} d r\right] \exp \left(2 \omega_{i}\right),
\end{gathered}
$$




$$
\begin{gathered}
T A N=\frac{1}{2\left(R-H_{l}\right)}\left[\mu_{g}\left(k^{2}\left(\phi_{g}^{\prime *} \phi_{g}+\phi_{g}^{*} \phi_{g}^{\prime}\right)+\left(\phi_{g}^{\prime \prime *} \phi_{g}^{\prime}+\phi_{g}^{\prime *} \phi_{g}^{\prime \prime}\right)-2 \frac{\left|\phi_{g}^{\prime}\right|^{2}}{R-H_{l}}\right)\right. \\
\left.-\mu_{l}\left(k^{2}\left(\phi_{l}^{\prime *} \phi_{l}+\phi_{l}^{*} \phi_{l}^{\prime}\right)+\left(\phi_{l}^{\prime \prime *} \phi_{l}^{\prime}+\phi_{l}^{\prime *} \phi_{l}^{\prime \prime}\right)-2 \frac{\left|\phi_{l}^{\prime}\right|^{2}}{R-H_{l}}\right)\right]_{r=R-H_{l}} \exp \left(2 \omega_{i}\right), \\
N O R=k^{2}\left|\phi_{g}\right|^{2} \frac{i \sigma}{k U_{i}-\omega} \frac{1}{\left(R-H_{l}\right)^{3}}\left(1-k^{2}\left(R-H_{l}\right)^{2}\right) \exp \left(2 \omega_{i}\right) .
\end{gathered}
$$

${ }^{1}$ A. H. Lefebvre, Atomization and Sprays (Hemisphere, New York, 1989).

${ }^{2}$ M. D. A. Lightfoot, S. A. Danczyk, and D. G. Talley, "A method to predict atomization performance in gas-centered swirl-coaxial injectors," Technical Paper No. AFRL-PR-ED-TP-2007-125 (Air Force Research Laboratory, 2007).

${ }^{3}$ M. D. A. Lightfoot, S. A. Danczyk, and D. G. Talley, "Atomization rate of gas-centered swirl-coaxial injectors," Technical Paper No. AFRL-RZ-ED-TP-2008-074 (Air Force Research Laboratory, 2008).

${ }^{4}$ M. D. A. Lightfoot, S. A. Danczyk, and D. G. Talley, "Scaling of gas-centered swirl-coaxial injectors," Technical Report No. ADA502809 (Air Force Research Laboratory, Edwards AFB, CA, 2008).

${ }^{5}$ J. Jeon, M. Hong, Y.-M. Han, and S. Y. Lee, "Experimental study on spray characteristics of gas-centered swirl coaxial injectors," Trans. ASME J. Fluid Eng. 133, 121303 (2011).

${ }^{6}$ B. Harper, J. Canino, S. D. Heister, and L. A. Carrison, "Hydrodynamic modelling of oxidizer-rich staged combustion injector flow," NASA Technical Report No. 20040075682, 2004.

${ }^{7}$ S. A. Schumaker, S. A. Danczyk, and M. D. A. Lightfoot, "Effect of swirl on gas-centered swirl-coaxial injectors," AIAA Paper 2011-5621, 2011.

${ }^{8}$ N. Otsu, "A threshold selection method from gray-level histograms," IEEE Trans. Syst., Man Cyber. 9, 62-66 (1979).

${ }^{9}$ J. Eggers and E. Villermaux, "Physics of liquid jets," Rep. Prog. Phys. 71, 036601 (2008).

${ }^{10}$ S. Marty, M. Hong, J.-P. Matas, A. Cartellier, and S. Y. Lee, "Spray characterization of gas-centered swirl coaxial injectors using an optical probe," in Proceedings of the 2011 Korean Society of Propulsion Engineers Fall Conference, KSPE, Busan, South Korea, 2011 (KSPE (Korea Society of Propulsion Engineers), 2011), pp. 172-177.

${ }^{11}$ Lord Rayleigh, "On the stability, or instability, of certain fluid motions," Proc. London Math. Soc. s1-11, 57 (1879).

${ }^{12}$ P. Marmottant and E. Villermaux, "On spray formation,” J. Fluid Mech. 498, 73 (2004).

${ }^{13}$ J.-P. Matas, S. Marty, and A. Cartellier, "Experimental and analytical study of the shear instability of a gas-liquid mixing layer," Phys. Fluids 23, 094112 (2011).

${ }^{14}$ P. E. Dimotakis, “Two-dimensional shear-layer entrainment,” AIAA J. 24, 1791-1796 (1986)

${ }^{15}$ E. Asmolov, "The inertial lift on a spherical particle in a plane Poiseuille flow at large channel Reynolds number," J. Fluid Mech. 381, 63-87 (1999).

${ }^{16}$ C. J. Heaton, "Linear instability of annular Poiseuille flow," J. Fluid Mech. 610, 391-406 (2008).

${ }^{17}$ T. Otto, M. Rossi, and T. Boeck, "Viscous instability of a sheared liquid-gas interface: Dependence on fluid properties and basic velocity profile," Phys. Fluids 25, 032103 (2013).

${ }^{18} \mathrm{M}$. Gaster, "A note on the relation between temporally-increasing and spatially-increasing disturbances in hydrodynamic stability," J. Fluid Mech. 14, 222 (1962).

${ }^{19}$ F. Charru and E. J. Hinch, "'Phase diagram' of interfacial instabilities in a two-layer Couette flow and mechanism of the long-wave instability," J. Fluid Mech. 414, 195-223 (2000).

${ }^{20}$ C. S. Yih, "Instability due to viscous stratification," J. Fluid Mech. 27, 337-352 (1967).

${ }^{21}$ A. P. Hooper and W. G. C. Boyd, "Shear-flow instability at the interface between two viscous fluids," J. Fluid Mech. 128, 507-528 (1983).

${ }^{22}$ A. P. Hooper and W. G. C. Boyd, "Shear-flow instability due to a wall and a viscosity discontinuity at the interface," J. Fluid Mech. 179, 201-225 (1987).

${ }^{23}$ E. J. Hinch, "A note on the mechanism of the instability at the interface between two fluids," J. Fluid Mech. 144, 463-465 (1984).

${ }^{24}$ T. Boeck and S. Zaleski, "Viscous versus inviscid instability of two-phase mixing layers with continuous velocity profile," Phys. Fluids 17, 032106 (2005).

${ }^{25}$ P. Boomkamp and R. Miesen, "Classification of instabilities in parallel two-phase flow," Int. J. Multiphase Flow 22, 67-88 (1996).

${ }^{26}$ D. Fuster, J.-P. Matas, S. Marty, S. Popinet, J. Hoepffner, A. Cartellier, and S. Zaleski, "Instability regimes in the primary breakup region of planar coflowing sheets," J. Fluid Mech. 736, 150-176 (2013).

${ }^{27}$ M. P. Juniper, "The effect of confinement on the stability of non-swirling round jet/wake flows," J. Fluid Mech. 605 , $227-252(2008)$. 